\title{
A Three-Dimensional Cement Hydration and Microstructure Program. I. Hydration Rate, Heat of Hydration, and Chemical Shrinkage
}

Dale P. Bentz

November 1995

Building and Fire Research Laboratory

National Institute of Standards and Technology

Gaithersburg, Maryland 20899

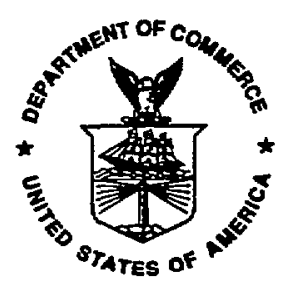

\section{U.S. Department of Commerce}

Ronald H. Brown, Secretary

Technology Administration

Mary L. Good, Under Secretary for Technology National Institute of Standards and Technology Arati Prabhakar, Director 



\begin{abstract}
A computer program that implements a three-dimensional model for the microstructural development occuring during the hydration of portland cement has been developed. The model includes reactions for the four major cement phases: tricalcium silicate, dicalcium silicate, tricalcium aluminate, and tetracalcium aluminoferrite, and the gypsum which is added to avoid flash setting. The basis for the computer model is a set of cellular automatalike rules for dissolution, diffusion, and reaction. The model operates on three-dimensional images of multi-phase cement particles generated to match specific characteristics of twodimensional images of real cements. To calibrate the kinetics of the model, experimental studies have been conducted at room temperature on two cements issued by the Cement and Concrete Reference Laboratory at NIST. Measurements of non-evaporable water content, heat of hydration, and chemical shrinkage over periods of up to 90 days have been performed for comparison with model predictions. The measurement of chemical shrinkage is particularly critical, as it allows an estimation of the density of the calcium silicate hydrate gel formed during the hydration to be made. The dispersion models of Knudsen have been applied in fitting both the model and experimental data. For the two cements investigated, it appears that a single function can be used to convert between model cycles and experimental time for the three water-to-cement ratios investigated in this study. This suggests that accurately capturing the particle size distribution, phase fractions, and phase distributions of a given cement allows for an accurate estimation of its hydration characteristics. Finally, the calibrated kinetic models for the two cements have been used to successfully predict 7 and 28-day compressive strengths of ASTM C109 $50 \mathrm{~mm}$ mortar cubes from 3-day compressive strength data, illustrating one engineering application for such a three-dimensional cement hydration and microstructure model.
\end{abstract}

Keywords: Building technology, cement hydration, chemical shrinkage, compressive strength, computer modelling, heat of hydration, microstructure, non-evaporable water, simulation. 



\section{Contents}

Abstract

List of Figures vii

List of Tables $\quad$ ix

Executive Summary

1 Introduction $\quad 1$

2 Experimental Techniques $\quad 1$

2.1 Materials and Cement Paste Preparation . . . . . . . . . . . . . . . . . 1

2.2 SEM and X-ray Imaging and Phase Separation . . . . . . . . . . . . . . . 3

2.3 Measurement of Particle Size Distribution . . . . . . . . . . . . . . . . 3

2.4 Non-Evaporable Water Content .................. 5

2.5 Heat of Hydration . . . . . . . . . . . . . . . . . . . . 7

2.6 Chemical Shrinkage . . . . . . . . . . . . . . . . 8

3 Computational Techniques $r$

3.1 Generation of a $3-\mathrm{D}$ Cement Particle Image $\ldots \ldots \ldots$

3.2 Distribution of Phases in a 3-D Cement Particle Image . . . . . . . . . . . 9

3.3 3-D Cement Hydration Model . . . . . . . . . . . . . . . . . . . . 10

3.4 Comments on Assumptions of the Hydration Model . . . . . . . . . . . 15

4 Results and Discussion $\quad 17$

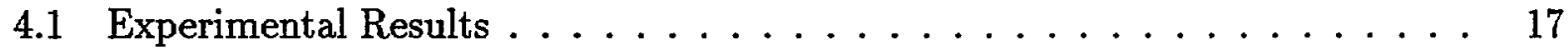

4.2 Model Results . . . . . . . . . . . . . . . . . . 20

4.3 Calibration of Model Using Experimental Results . . . . . . . . . . . 23

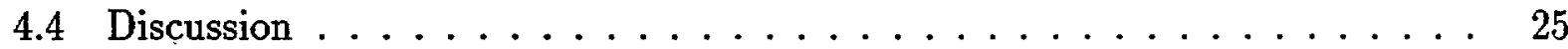

5 Conclusions $\quad 28$

6 Acknowledgments $\quad 28$

7 References $\quad 29$

A Graphs for Fit of Knudsen's Dispersion Models to Experimental Results 32

B Model vs. Experimental Results $\quad 36$

C Variability of Computer Simulation Results $\quad 47$ 



\section{List of Figures}

1 Flow diagram summarizing experimental and modelling program for predicting cement performance. . . . . . . . . . . . . . . . .

2 Final segmented two-dimensional image of CCRL Cement 115. Phases from brightest to darkest are: $C_{3} A$, gypsum, $C_{4} A F, C_{3} S, C_{2} S$, and porosity. Image is approximately $250(\mu \mathrm{m}) \times 200 \mu \mathrm{m} . \ldots \ldots \ldots \ldots \ldots$

3 Final segmented two-dimensional image of CCRL Cement 116. Greylevel assignments are the same as in Figure $2 \ldots \ldots \ldots \ldots \ldots$

4 Experimental heat release signal vs. time. . . . . . . . . . . . . .

5 Portion of initial 3-D image of Cement 116 with $w / c=0.4$. Phases from brightest to darkest are: $C_{3} A$, gypsum, $C_{4} A F, C_{3} S, C_{2} S$, and porosity. . . .

6 Cement model reactions - numbers below reactions indicate volume stoichiometries. . . . . . . . . . . . . . . . .

7 State transition diagram for 3-D Cement Hydration Model. Arrow patterns denote the collision of two species to form a hydration product. $\mathrm{f}([\mathrm{X}])$ denotes that nucleation or dissolution probability is a function of concentration or volume fraction of phase $\mathrm{X}$.

8 Experimental results for CCRL Cements and 115 and 116 vs. time.

9 Fits of Knudsen's dispersion models to experimental non-evaporable water content ( $\mathrm{g}_{2} \mathrm{O} / \mathrm{g}$ cement) vs. time. . . . . . . . . . . . . . . . .

10 Model anhydrous cement volume fractions vs. elapsed cycles for Cement 116 with $w / c=0.4 \ldots \ldots \ldots \ldots \ldots \ldots \ldots \ldots \ldots \ldots$

11 Model porosity and reaction product volume fractions vs. elapsed cycles for Cement 116 with $w / c=0.4 \ldots \ldots \ldots \ldots \ldots \ldots$

12 Model aluminate reaction product volume fractions vs. elapsed cycles for $\mathrm{Ce}$ ment 116 with $w / c=0.4$.

13 Comparison of heat release via major phase enthalpies and heats of formation for Cement 116 with $w / c=0.45$.

14 Predicted and measured compressive strength development for Cement 115. .

15 Predicted and measured compressive strength development for Cement 116. .

16 Fits of Knudsen's dispersion models to experimental non-evaporable water content vs. time for Cement 115 and $w / c=0.3 . \ldots \ldots \ldots \ldots \ldots$

17 Fits of Knudsen's dispersion models to experimental non-evaporable water content vs. time for Cement 115 and $w / c=0.4 \ldots \ldots \ldots \ldots \ldots$

18 Fits of Knudsen's dispersion models to experimental non-evaporable water content vs. time for Cement 115 and $w / c=0.45 . \ldots \ldots \ldots \ldots \ldots$

19 Fits of Knudsen's dispersion models to experimental non-evaporable water content vs. time for Cement 116 and $w / c=0.3$.

20 Fits of Knudsen's dispersion models to experimental non-evaporable water content vs. time for Cement 116 and $w / c=0.4 \ldots \ldots \ldots \ldots \ldots$

21 Fits of Knudsen's dispersion models to experimental non-evaporable water content vs. time for Cement 116 and $w / c=0.45 . \ldots \ldots \ldots \ldots$

22 Measured and model degree of hydration vs. time for Cement 115 with $w / c=$

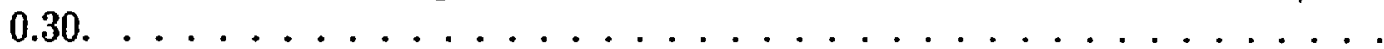

23 Measured and model chemical shrinkage vs. time for Cement 115 with $w / c=$ 0.30 . 
24 Measured and model degree of hydration vs. time for Cement 115 with $w / c=$

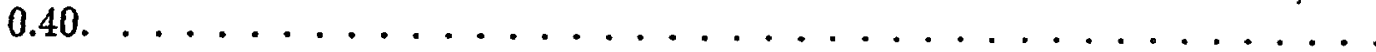

25 Measured and model chemical shrinkage vs. time for Cement 115 with $w / c=$

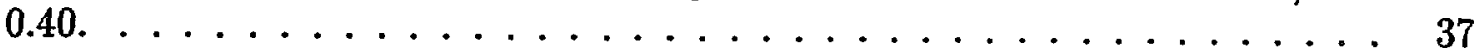

26 Measured and model heat release vs. time for Cement 115 with $w / c=0.40$. . 38

27 Measured and model heat release vs. time for Cement 115 with $w / c=0.45$. . 38

28 Measured and model degree of hydration vs. time for Cement 115 with $w / c=$

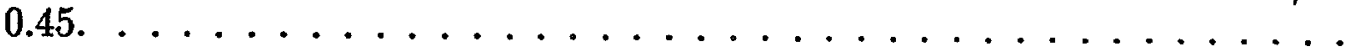

29 Measured and model chemical shrinkage vs. time for Cement 115 with $w / c=$

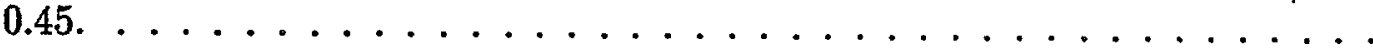

30 Measured and model degree of hydration vs. time for Cement 116 with $w / c=$

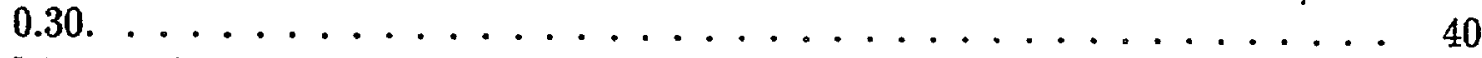

31 Measured and model chemical shrinkage vs. time for Cement 116 with $w / c=$

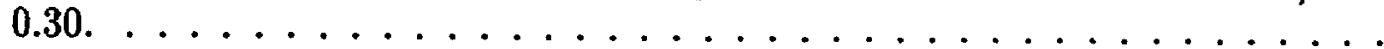

32 Measured and model degree of hydration vs. time for Cement 116 with $w / c=$ $0.40 . \ldots \ldots \ldots \ldots \ldots \ldots \ldots \ldots \ldots \ldots$

33 Measured and model chemical shrinkage vs. time for Cement 116 with $w / c=$

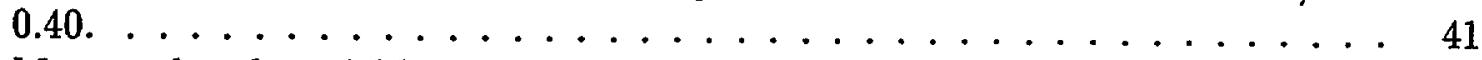

34 Measured and model heat release vs. time for Cement 116 with $w / c=0.40$. .

35 Measured and model heat release vs. time for Cement 116 with $w / c=0.45$. .

36 Measured and model degree of hydration vs. time for Cement 116 with $w / c=$ $0.45 . \ldots \ldots \ldots \ldots \ldots \ldots \ldots \ldots \ldots$

37 Measured and model chemical shrinkage vs. time for Cement 116 with $w / c=$

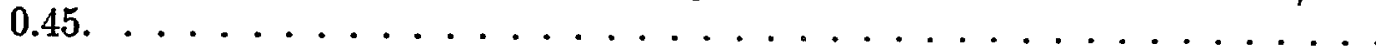

38 Measured and model degree of hydration vs. time for Cement 115 with $w / c=$ 0.40 for monophase Bogue representation. . . . . . . . . . . .

39 Measured and model chemical shrinkage vs. time for Cement 115 with $w / c=$ 0.40 for monophase Bogue representation. . . . . . . . . . . . . .

40 Measured and model heat release vs. time for Cement 115 with $w / c=0.40$ for monophase Bogue representation. . . . . . . . . . . . . .

41 Measured and model degree of hydration vs. time for Cement 116 with $w / c=$ 0.40 for monophase Bogue representation. . . . . . . . . . . . .

42 Measured and model chemical shrinkage vs. time for Cement 116 with $w / c=$ 0.40 for monophase Bogue representation. . . . . . . . . . . 46

43 Measured and model heat release vs. time for Cement 116 with $w / c=0.40$ for monophase Bogue representation. . . . . . . . . . . . . 46

44 Variation in results for three executions of 3-D hydration model on a single starting microstructure (Cement 116 with $w / c=0.4$ ). . . . . . . . 47

45 Variation in results for single executions of 3-D hydration model on three different starting microstructures (Cement 116 with $w / c=0.4$ ). . . . . 47 


\section{List of Tables}

1 Phase Fractions for CCRL Cement $115 \ldots \ldots \ldots \ldots$

2 Phase Fractions for CCRL Cement $116 \ldots \ldots \ldots \ldots$

3 Discretized PSDs for CCRL Cements 115 and $116 \ldots \ldots \ldots 6$

4 Measured Specific Surface Areas for CCRL Cements 115 and 116 . . . . . 6

5 Non-evaporable Water Contents for Major Phases of Cement . . . . . . . . . 7

6 Physical Properties of Cementitious Materials . . . . . . . . . . . . . . 13

7 Enthalpy of Complete Hydration for Major Phases of Cement . . . . . . . 15

8 Measured Seven Day Heats of Hydration for CCRL Cements 115 and 116 . . 19

9 Parameters for Knudsen's Linear Dispersion Model for Cements 115 and 11621

10 Parameters for Knudsen's Parabolic Dispersion Model for Cements 115 and 11621

11 Parameter for Converting Cycles to Time for Cements 115 and $116 \ldots 25$ 



\section{EXECUTIVE SUMMARY}

A three-dimensional hydration and microstructure model for portland cement has been developed and preliminarily validated against experimental data. For input, the model requires the particle size distribution and a set of SEM/X-ray images for the cement of interest. With this information, a three-dimensional representation of the cement particles in water is constructed which matches the following characteristics of the input information: 1) the particle size distribution, 2) the individual phase volume fractions, and 3) the individual phase surface fractions which are in contact with porosity (water). Additionally, the cement particles may be either flocculated or dispersed during this construction process to better represent real cement-water systems.

Starting with the constructed three-dimensional cement particle image, a computer model based on a set of cellular-automata-like rules has been developed for simulating the hydration reactions that occur between cement and water. The model accounts for the major reactions of the cement clinker phases (tricalcium silicate, dicalcium silicate, tricalcium aluminate, and tetracalcium aluminoferrite) and gypsum. Reaction stoichiometries and reactant and product physical properties (molar volume, density, and heat of formation) have been taken from the available literature or obtained from calibration against experimental data. In addition to representing the microstructural evolution which occurs during hydration, the model also provides quantitative information on the amount of hydration which has occurred, the heat which would be released under isothermal conditions, and the amount of chemical shrinkage which would occur.

To validate the model, experimental studies have been conducted on Cements 115 and 116 issued in 1995 by the Cement and Concrete Reference Laboratory (CCRL) at NIST. At $25^{\circ} \mathrm{C}$, non-evaporable water content, heat release via isothermal microcalorimetry, and chemical shrinkage have been measured at three different $w / c$ ratios $(0.3,0.4$, and 0.45$)$. The first of these can be converted to a degree of hydration by normalization by the value measured for fully hydrated samples of each of the two cements. The three experimental measurements exhibit good agreement with one another over the range of $w / c$ ratios studied. The kinetic (dispersion) models of Knudsen have been utilized to fit the non-evaporable water content vs. time for times up to 90 days. The parabolic dispersion model has been found to provide the best overall fit to the experimental data, with a relatively constant induction time for the two cements and three $w / c$ ratios.

Using the fitted parabolic dispersion models, the model results have been calibrated to the experimental data. For Cements 115 and 116, a single set of parameters can be used to relate model cycles to real time via an equation of the form: time $=t_{0}+B *$ cycles $^{2}$. With this calibration, the agreement between model and experimental degrees of hydration, heat releases, and chemical shrinkages is in general excellent. In adddition, based on the gelspace ratio theory of Powers and Brownyard, the hydration model has been used to predict the 7 and 28-day compressive strengths of ASTM C109 mortar cubes from the measured 3 -day strengths and the calibrated hydration kinetics. The predictions have been found to lie well within the standard deviation of the CCRL interlaboratory testing program, suggesting one promising engineering application of the three-dimensional cement hydration and microstructure program.

In the future, efforts will concentrate on extending these results to other temperatures and calibrating the incorporation of silica fume into the microstructure model. 



\section{Introduction}

Although often taken for granted as a commodity material, portland cement is a complex heterogeneous particulate material. When mixed with water, a variety of reactions transform the initial suspension into a rigid, load-bearing matrix which comprises the binder phase of a typical concrete. To this date, precise knowledge of the mechanisms, stoichiometries, thermodynamics, and kinetics of the hydration reactions remains to be provided. With this state of affairs, it is often difficult to quantitatively relate the microstructure of cement paste to its ultimate properties such as strength, diffusivity, and permeability, so that material performance may be improved. The ability to accurately predict performance will play a key role in the ongoing paradigm shift from prescriptive to performance-based standards [1].

In recent years, computer modelling has been successfully applied to elucidating microstructure-property relationships of cement-based materials [2]. Such elucidation requires a two-step process: generation of a representative microstructure in the computer, and computation of the property of interest, often using finite difference or finite element techniques. Although much information has been obtained using a three-dimensional microstructure model based solely on the hydration of the predominant phase present in portland cement, tricalcium silicate $\left(C_{3} S^{1}\right)$ [3, 4], many problems of interest to cement researchers require a microstructural representation which includes all of the major phases of portland cement. Thus, recent efforts have focused on developing a three-dimensional cement hydration and microstructure program which accounts for the multi-size and multiphase nature of cement grains.

Computationally, this requires acquisition or generation of a representative three-dimensional starting microstructure for use as input into the hydration and microstructure development program. Here, computational techniques are developed for converting a set of two-dimensional scanning electron microscopy (SEM) images into a three-dimensional representation of a given cement. The developed procedures reproduce the particle size distribution of the cement as well as the individual phase volume and surface area fractions. The final 3-D image is then used as input for the cellular automata-based hydration model. In addition to providing a 3-D map of the microstructure as it evolves, the hydration code also outputs the degree of hydration, the heat released, and the chemical shrinkage as a function of the number of hydration "cycles" which have been executed. These three model measures can be compared against their experimental counterparts to calibrate and validate the kinetics of the cement hydration model. The model and experimental program are summarized in the flow diagram in Figure 1.

\section{Experimental Techniques}

\subsection{Materials and Cement Paste Preparation}

As part of its proficiency sample program, the ASTM-sponsored Cement and Concrete Reference Laboratory (CCRL), located at NIST, issues two portland cements semi-annually for testing by the appropriate ASTM chemical and physical test methods by participating laboratories [5]. CCRL issued Cements 115 and 116 in January of 1995. A sufficient supply of these cements stored in a double layer of plastic in cardboard boxes was obtained for

\footnotetext{
${ }^{1}$ Conventional cement chemistry notation is used throughout this report with $\mathrm{C}=\mathrm{CaO}, \mathrm{S}=\mathrm{SiO}_{2}, \mathrm{~A}=$ $\mathrm{Al}_{2} \mathrm{O}_{3}, \mathrm{~F}=\mathrm{Fe}_{2} \mathrm{O}_{3}, \mathrm{H}=\mathrm{H}_{2} \mathrm{O}$, and $\overline{\mathrm{S}}=\mathrm{SO}_{3}$
} 


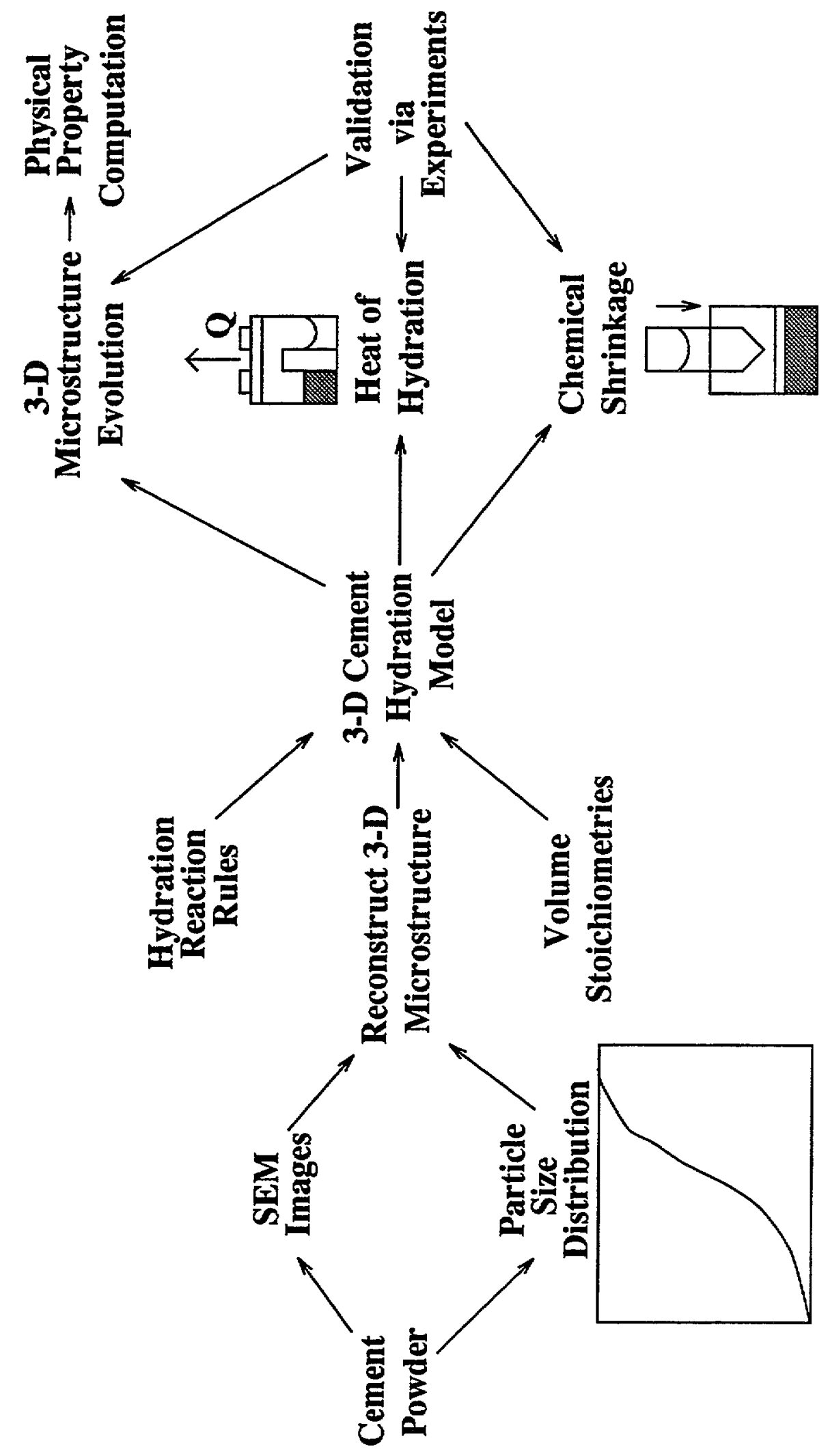

Figure 1: Flow diagram summarizing experimental and modelling program for predicting cement performance. 
the present studies. Results [5], obtained using the appropriate ASTM [6] test method, and available in the CCRL report, include the cements' oxide compositions (ASTM C114), the initial and final times of set via the Vicat (ASTM C191) and Gillmore (ASTM C266) needle methods, measured finenesses (ASTM C204 and C115), mortar cube compressive strengths (ASTM C109), and the heats of hydration at 7 and 28 days of age measured via the heat of solution method (ASTM C186).

For the non-evaporable water content and chemical shrinkage studies described below, cement pastes were prepared with water-to-cement $(w / c)$ ratios of $0.3,0.4$, and 0.45 . The cement powder and necessary mass of water were mixed together by kneading by hand in a sealed plastic bag for two to three minutes. Samples were then removed and stored in capped plastic vials and small glass jars for the non-evaporable water content and chemical shrinkage measurements, respectively. In both cases, after placing the cement paste sample (typically 10-15 grams) in its container, about $1 \mathrm{~mL}$ of water was added on top of the cement paste to maintain saturated conditions throughout the experiment. For the non-evaporable water content measurement, the samples were stored at $25^{\circ} \mathrm{C}$ until being evaluated. Evaluations of non-evaporable water content were typically made after the following times of hydration: 8 hours and $1,2,3,7,14,28,56$, and 90 days.

\subsection{SEM and X-ray Imaging and Phase Separation}

The experimental and image processing techniques which have been developed for segmenting two-dimensional images of cements into their five major phases $\left(C_{3} S, C_{2} S, C_{3} A, C_{4} A F\right.$, and gypsum) have been described in detail in [7]. The cement of interest is dispersed in a low viscosity epoxy which is subsequently cured. A polished surface is prepared and viewed in the scanning electron microscope. Based on the signal intensity of the backscattered electron image and the intensities of the $\mathrm{X}$-ray images for $\mathrm{Ca}, \mathrm{Si}, \mathrm{Al}, \mathrm{Fe}$, and $\mathrm{S}$, each pixel in the two-dimensional image can be identified as either porosity or one of the five phases of portland cement. For example, strong signals for iron, aluminum, and calcium indicate the presence of the $C_{4} A F$ phase. Once an initial segmentation is performed, a type of median filtering is applied to remove some of the random noise present after the segmentation process. Here, each non-porosity pixel is replaced by the majority solid phase present in a limited neighborhood (e.g., $3 \times 3$ ) centered at the pixel. The final images are then analyzed to determine the area fraction of each phase and the perimeter fraction of each phase in contact with porosity. Images of the two CCRL cements analyzed in this project using these procedures are provided in Figures 2 and 3. Tables 1 and 2 provide the measured area and perimeter phase fractions along with the phase fractions calculated from the reported oxide compositions [5] using the conventional Bogue calculation [8].

\subsection{Measurement of Particle Size Distribution}

The particle size distributions (PSDs) for Cements 115 and 116 were measured at the University of Illinois using an x-ray sediograph technique. The result from the test is a curve of cumulative mass fraction smaller than an equivalent spherical diameter. This curve is discretized (binned) into $2 \mu \mathrm{m}$ increments for use in the three-dimensional cement hydration model. The discretized PSDs determined for Cements 115 and 116 and used in the computer modelling are provided in Table 3. The calculated surface areas along with those measured [5] using the available ASTM techniques [6], are provided in Table 4. While the 


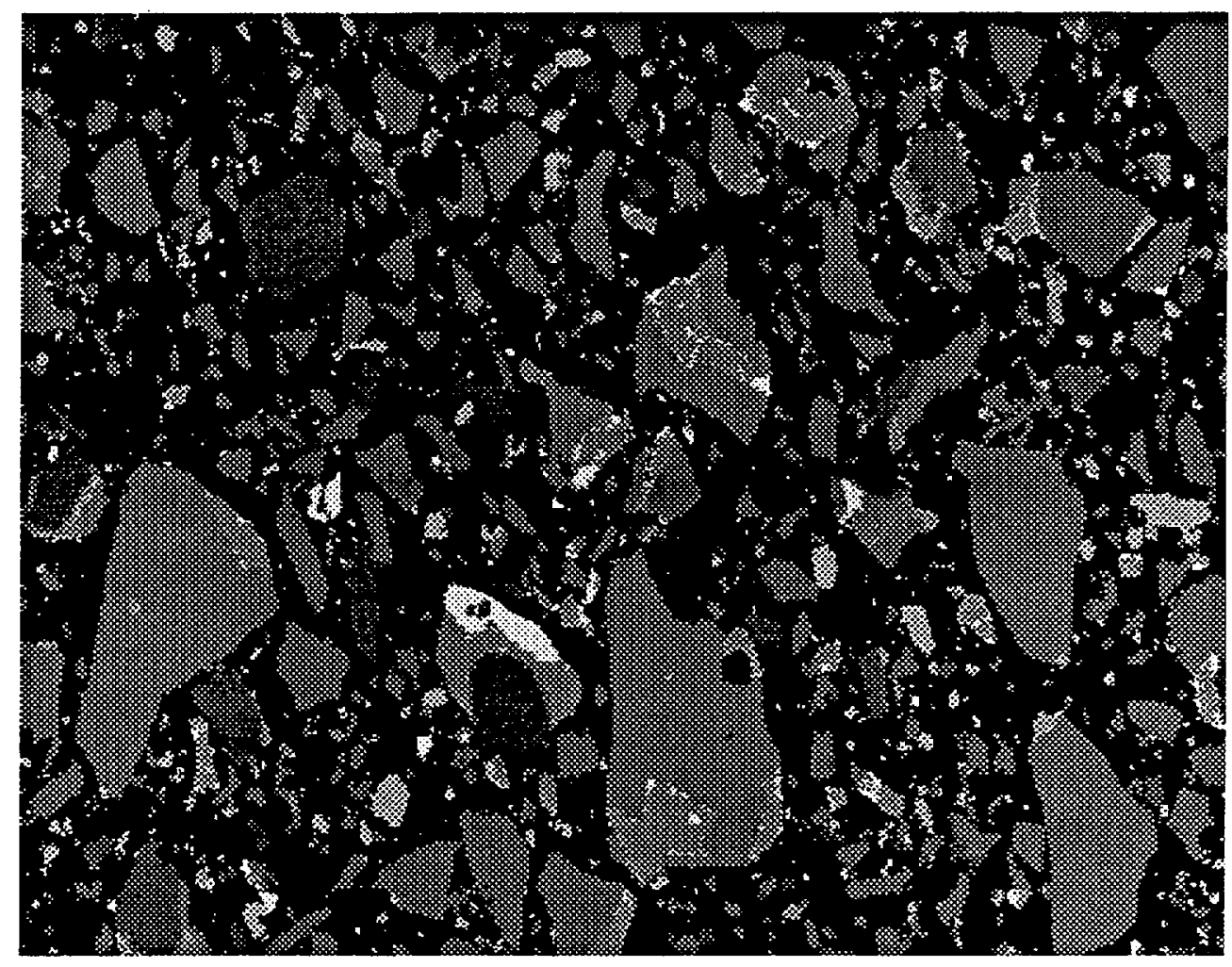

Figure 2: Final segmented two-dimensional image of CCRL Cement 115. Phases from brightest to darkest are: $C_{3} A$, gypsum, $C_{4} A F, C_{3} S, C_{2} S$, and porosity. Image is approximately $250(\mu \mathrm{m}) \times 200 \mu \mathrm{m}$.

experimentally measured surfaces areas are seen to be similar although differing between the two techniques, according to the PSD measurement, Cement 116, with an average particle radius of $4.8 \mu \mathrm{m}$ on a mass basis, is finer than Cement 115 , with an average particle radius of $6.1 \mu \mathrm{m}$. The surface areas calculated based on the PSDs given in Table 3 are lower than the experimental values due to both the truncation of the PSD (i.e., the elimination of particles smaller than $3 \mu \mathrm{m}$ ) and the assumption of spherical particle shapes.

Table 1: Phase Fractions for CCRL Cement 115

\begin{tabular}{cccc}
\hline Phase & Perimeter fraction & Area fraction & Bogue volume fraction \\
\hline & & & \\
$C_{3} S$ & 0.504 & 0.605 & 0.547 \\
$C_{2} S$ & 0.239 & 0.221 & 0.214 \\
$C_{3} A$ & 0.045 & 0.032 & 0.081 \\
$C_{4} A F$ & 0.076 & 0.097 & 0.093 \\
Gypsum & 0.136 & 0.046 & 0.065 \\
\hline
\end{tabular}




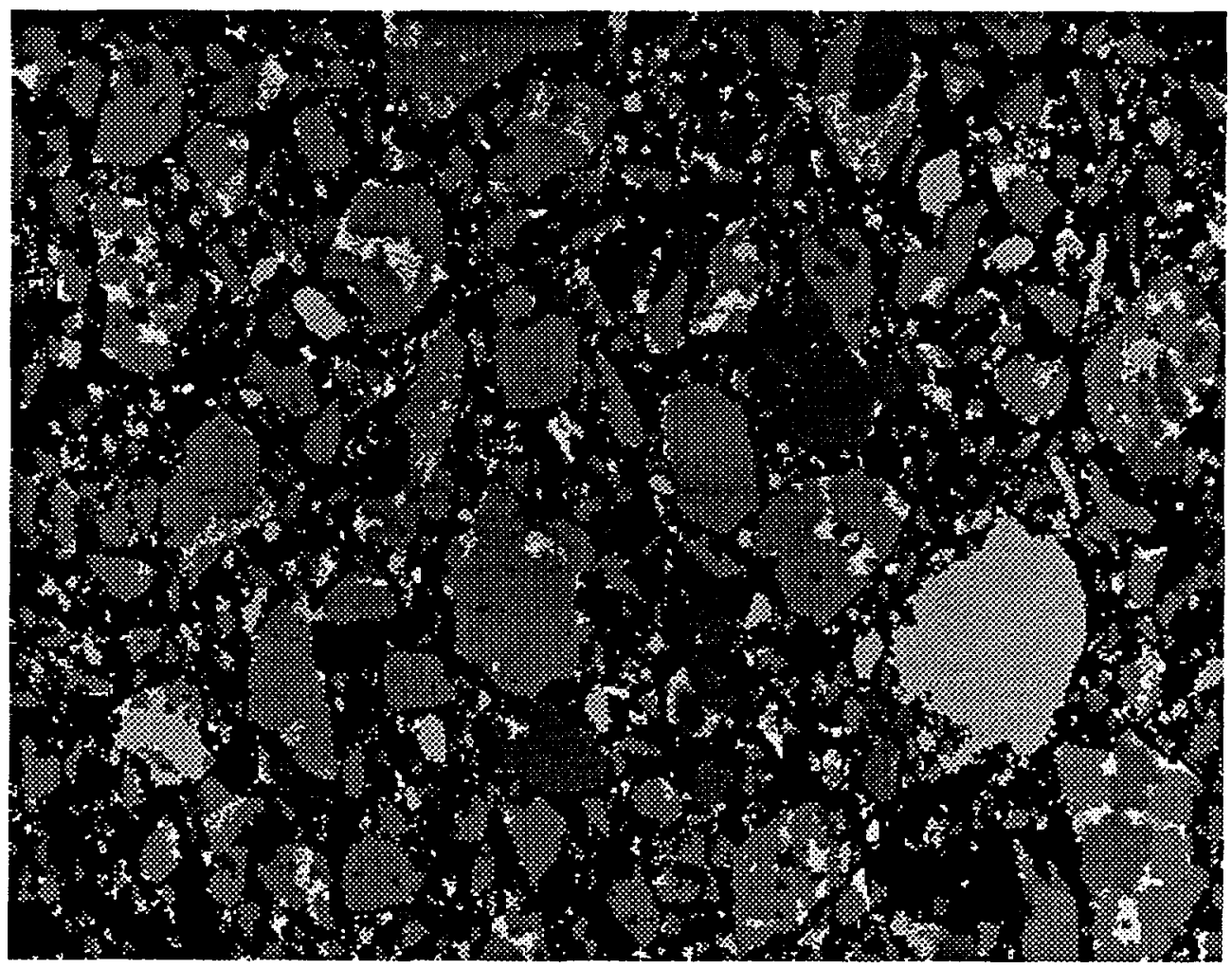

Figure 3: Final segmented two-dimensional image of CCRL Cement 116. Greylevel assignments are the same as in Figure 2.

Table 2: Phase Fractions for CCRL Cement 116

\begin{tabular}{cccc}
\hline Phase & Perimeter fraction & Area fraction & Bogue volume fraction \\
\hline & & & \\
$C_{3} S$ & 0.399 & 0.575 & 0.544 \\
$C_{2} S$ & 0.242 & 0.190 & 0.195 \\
$C_{3} A$ & 0.084 & 0.061 & 0.137 \\
$C_{4} A F$ & 0.020 & 0.031 & 0.054 \\
Gypsum & 0.255 & 0.142 & 0.071 \\
\hline
\end{tabular}

\subsection{Non-Evaporable Water Content}

After achieving the required age, samples for the non-evaporable water content, $W_{N}$, determination were ground to a powder using a mortar and pestle and flushed with methanol, using a porous ceramic filter and a vacuum, to stop the hydration. The resultant powder was divided approximately in half and placed in two crucibles of known mass and left overnight (on the order of 20 hours) in an oven at $105^{\circ} \mathrm{C}$. When removed from the oven, the mass of the crucibles and samples were redetermined before placing them in a furnace at $950^{\circ} \mathrm{C}$ for 
Table 3: Discretized PSDs for CCRL Cements 115 and 116

\begin{tabular}{lll}
\hline Diameter $(\mu \mathrm{m})$ & $\begin{array}{c}\text { Weight Fraction } \\
\text { Cement 115 }\end{array}$ & $\begin{array}{c}\text { Weight Fraction } \\
\text { Cement 116 }\end{array}$ \\
\hline & & \\
3 & 0.162 & 0.245 \\
5 & 0.136 & 0.153 \\
7 & 0.125 & 0.122 \\
9 & 0.075 & 0.087 \\
11 & 0.095 & 0.107 \\
13 & 0.064 & 0.077 \\
15 & 0.056 & 0.038 \\
17 & 0.053 & 0.036 \\
19 & 0.044 & 0.039 \\
21 & 0.041 & 0.046 \\
23 & 0.036 & 0.005 \\
25 & 0.036 & 0.023 \\
27 & 0.021 & 0.000 \\
29 & 0.015 & 0.004 \\
31 & 0.015 & 0.013 \\
33 & 0.012 & 0.005 \\
35 & 0.003 & 0.000 \\
37 & 0.010 & 0.000 \\
\hline
\end{tabular}

a minimum of four hours. The non-evaporable water content was calculated as the average difference between the 950 and $105^{\circ} \mathrm{C}$ mass measurements for the two crucibles, corrected for the loss on ignition of the cement powder itself, which was assessed in a separate crucible experiment.

To convert the non-evaporable water measurements to estimated degrees of hydration, $\alpha$, it was necessary to determine the non-evaporable water content for a fully hydrated sample. Cement paste samples at a $w / c=3.0$ were prepared for each of the two cements. The samples were each continuously ground in a jar mill, containing approximately $50 \%$ volume fraction of stainless steel balls, for a period exceeding 28 days. Samples were removed periodically and the non-evaporable water content assessed using the above procedure. Little change in the non-evaporable water content was observed after the first seven days of grinding. For Cement 115, a value of $0.226 \mathrm{~g} \mathrm{H}_{2} \mathrm{O} / \mathrm{g}$ cement was determined at "complete" hydration,

Table 4: Measured Specific Surface Areas for CCRL Cements 115 and 116

\begin{tabular}{cccc}
\hline Cement & $\begin{array}{c}\text { Air permeability } \\
\text { Surface Area }\left(\mathrm{cm}^{2} / \mathrm{g}\right)[\mathrm{g}]\end{array}$ & $\begin{array}{c}\text { Wagner Turbidimeter } \\
\text { Surface Area }\left(\mathrm{cm}^{2} / \mathrm{g}\right)[5]\end{array}$ & $\begin{array}{c}\text { PSD Calculated } \\
\text { Surface Area }\left(\mathrm{cm}^{2} / \mathrm{g}\right)\end{array}$ \\
\hline & & & \\
115 & 3633 & 2061 & 838 \\
116 & 3646 & 1931 & 1012 \\
\hline
\end{tabular}


which can be compared to a value of 0.240 calculated based on the Bogue composition of the cement and tabulated values for the non-evaporable water content of the major cement phases [9] provided in Table 5. For Cement 116, values of 0.235 and 0.239 were determined using the experimental procedure and direct calculation, respectively.

Table 5: Non-evaporable Water Contents for Major Phases of Cement

\begin{tabular}{ccc}
\hline Phase & $\begin{array}{c}\text { Coefficient } \\
\text { g water/g cement }\end{array}$ & Source \\
\hline$C_{3} S$ & 0.24 & {$[9]$} \\
$C_{2} S$ & 0.21 & {$[9]$} \\
$C_{3} A$ & 0.40 & {$[9]$} \\
$C_{4} A F$ & 0.37 & {$[9]$} \\
Free lime & 0.33 & Direct calculation ${ }^{a}$ \\
\hline
\end{tabular}

${ }^{a}$ Assuming reaction of free lime with water to produce portlandite (CH).

\subsection{Heat of Hydration}

The heats of hydration of the two cements were assessed using a multi-chambered microcalorimeter constructed at NIST [10]. A known mass of cement, along with several small stainless steel balls to facilitate mixing, were placed in a sealed calorimetric cell which was then equilibrated in the main calorimeter chamber. After a steady heat flux signal was obtained, the cell was removed, the appropriate mass of water (also thermally equilibrated to the calorimeter temperature) quickly added using a syringe, and hand mixing performed (by shaking the cell) before restoring the cell to the calorimeter chamber. The voltage signals produced (proportional to heat flux) by the calorimeter cells were digitized using a PC-based high resolution A/D data acquisition system. Thus, during the initial hydration, data could be taken at 30 second intervals. Once the reactions slowed, data was typically acquired every 5 or 10 minutes over a period of at least 7 days. At longer times, the signal of the calorimeter is very close to its background level, so that detection of the slow but ongoing hydration becomes unreliable. In analyzing the heat release data, the initial exothermic "mixing" peak was ignored due to the necessity of removing the sample cell from the calorimetric chamber to assure adequate mixing. This could result in a difference in the cumulative heat released over a period of 7 days on the order of $10 \mathrm{~kJ} / \mathrm{kg}$ or about $4 \%$, as estimated from samples mixed in situ in the calorimeter. Due to the mixing difficulties at low $w / c$ ratios, calorimetric measurements were only performed at the two higher $w / c$ ratios of 0.4 and 0.45 .

Figure 4 provides a sample plot of the obtained signal for Cement 116 at $25^{\circ} \mathrm{C}$ for $w / c=$ 0.4 for the first 24 hours of data acquisition. This signal vs. time was then numerically integrated to obtain the cumulative heat release $(\mathrm{kJ} / \mathrm{kg}$ cement) vs. time curves which will be presented in the results. 


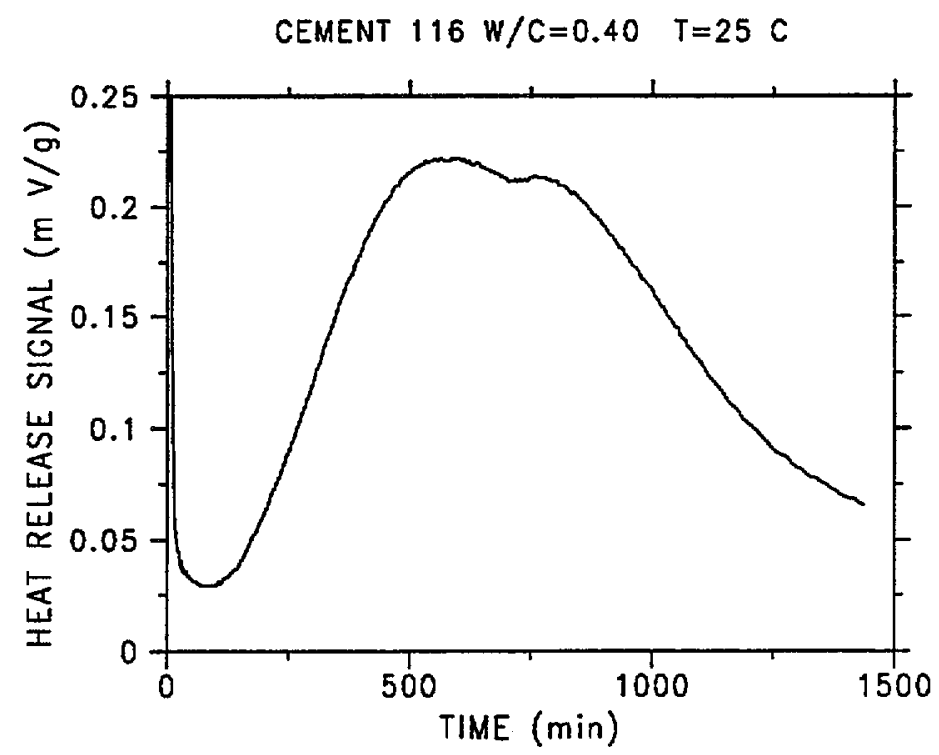

Figure 4: Experimental heat release signal vs. time.

\subsection{Chemical Shrinkage}

Chemical shrinkage, the volume reduction associated with the reaction between cement and water in hydrating cement paste, was assessed using the method described by Geiker [11], which is similar to that recently employed by Tazawa et al. [12]. While the latter authors concluded that chemical shrinkage is directly proportional to degree of hydration, they further stated that chemical shrinkage is not directly related to autogenous or self-desiccation shrinkage. Conversely, Hua et al. $[13,14]$ have recently established a model which successfully directly relates autogenous shrinkage to the capillary pressures induced by chemical shrinkage. Thus, measurements of chemical shrinkage may serve a dual purpose, quantification of hydration rates and indication of system susceptibility to self-desiccation shrinkage.

To assess chemical shrinkage, a known mass of cement paste (typically $10 \mathrm{~g}$ ) was placed in the bottom of a small glass jar, with a diameter of $2.5 \mathrm{~cm}$ and a height of about $6 \mathrm{~cm}$. After covering the cement paste with about $1 \mathrm{~mL}$ of water, the remainder of the jar was filled with an hydraulic oil. The jar was then sealed with a rubber stopper encasing a pipette graduated in $0.01 \mathrm{~mL}$ increments. The jar was then placed in a constant temperature water bath $\left(\mathrm{T}=25^{\circ} \mathrm{C}\right)$ and the oil level monitored to the nearest $0.0025 \mathrm{~mL}$ over time. A control sample using only cement powder and oil (no water) was used to correct for minor room temperature fluctuations. By normalizing the change in volume by the mass of cement in the sample, the chemical shrinkage per gram of initial cement ( $\mathrm{mL} / \mathrm{g}$ cement) could be determined. In all cases, two specimens were run for each $w / c$ ratio and cement, with the average result being reported.

\section{Computational Techniques}

\subsection{Generation of a 3-D Cement Particle Image}

The first step in generating an original three-dimensional cement particle microstructure is the creation of a three-dimensional image of digitized spheres representing the actual PSD 
of the cement of interest. Spheres have been chosen for computational simplicity, although digitized ellipsoidal shapes could also be employed. Bonen and Diamond [15] have measured the aspect ratio of cement particles in 2-D SEM images and found values on the order of two, suggesting that the use of spheres is an adequate approximation. For this study, a computational volume $100 \times 100 \times 100$ pixels is typically employed. Spherical particles following the measured PSD are placed into this computational volume from largest to smallest in diameter, such that no two particles overlap. Periodic boundaries [3] are used to eliminate edge effects; if a portion of a particle extends beyond one or more faces of the 3-D box, the remainder of its volume is protruded into the opposite face. Particles typically range from 3 to 35 pixels in diameter. Since the scale of the model is such that one pixel is equivalent to $1 \mu \mathrm{m}$, the cement particles range from $3(\mu \mathrm{m})$ to $35 \mu \mathrm{m}$ in diameter, which encompasses most of the PSD of a typical cement, although some truncation at both the high and low ends is necessary.

Phase assignment during particle placement is implemented in one of two manners. In the first case, the particles are randomly assigned to be one of the major cement phases to match the volumetric phase fractions computed from the calculated Bogue composition of the cement. In this case, the particles are monophase and no effort is made to utilize the information available from the SEM image analysis of the cement. Thus, the only required inputs are the PSD of the cement and its Bogue potential phase composition. In the second case, during this initial placement, a portion of the particles are assigned to be gypsum, based on the Bogue calculation for the cement, with the remainder being cement. The four major clinker phases are then distributed amongst the pixels assigned to be cement as described in the next section, in order to match the volumetric and surface phase fractions determined from the SEM images.

During particle placement, particles can be optionally flocculated or dispersed [16]. For this study, since no dispersing agents were used in the experimental procedures, the particles were totally flocculated. To do this, each particle centroid is displaced a distance of one pixel in one of six random directions $( \pm x, \pm y, \pm z)$. If this move causes the current particle to impact another one, the two are flocculated and move as a single unit in all future random displacements. This algorithm is repetitively implemented until the user-selected number of flocs (one single floc in this study) is formed. The use of this algorithm is justified by recent experimental results [17] suggesting that such a flocculated structure will reform even after mixing.

\subsection{Distribution of Phases in a 3-D Cement Particle Image}

Once the particles are placed, the next step is to create multi-phase particles by distributing the phases is such a manner as to match the volume and surface area fractions as estimated from the two-dimensional SEM images. A modification of a technique employed to reconstruct three-dimensional porous media from a two-dimensional image $[18,19]$ is used for this purpose. To begin, the two point correlation function is determined for three different phase combinations in the two-dimensional segmented SEM image: the combined silicates $\left(C_{3} S\right.$ and $C_{2} S$ ), the $C_{3} S$, and either the $C_{3} A$ or the $C_{4} A F$ (whichever is the more abundant of the two). This function is evaluated for an $\mathrm{M} \times \mathrm{N}$ image using the following equation:

$$
S(x, y)=\sum_{i=1}^{M-x} \sum_{j=1}^{N-y} \frac{I(i, j) \times I(i+x, j+y)}{(M-x) \times(N-y)}
$$


where $I(x, y)$ is one if the pixel at location $(x, y)$ contains the phase(s) of interest and zero otherwise. These values are then converted to $S(r)$ for distances $\mathbf{r}$ in pixels by:

$$
S(r)=\frac{1}{2 r+1} \sum_{l=0}^{2 r} S\left(r, \frac{\pi l}{4 r}\right)
$$

where $S(r, \theta)=S(r \cos \theta, r \sin \theta)$ is obtained by bilinear interpolation from the values of $S(x, y)$.

The two-point correlation function for the $C_{3} S$ and $C_{2} S$ is used to separate the cement particles into silicates and aluminates. To do this, each pixel in the three-dimensional cement particle image is assigned a random number following a normal distribution, $N(x, y, z)$, generated using the Box-Muller method [20]. This random number image is then filtered using the autocorrelation function, $F(x, y, z)$ :

$$
F(r)=F(x, y, z)=\frac{\left[S\left(r=\sqrt{x^{2}+y^{2}+z^{2}}\right)-S(0) \times S(0)\right]}{[S(0)-S(0) \times S(0)]}
$$

The resultant image, $R(x, y, z)$, is calculated as:

$$
R(x, y, z)=\sum_{i=0}^{30} \sum_{j=0}^{30} \sum_{k=0}^{30} N(x+i, y+j, z+k) \times F(i, j, k)
$$

Finally, for those pixels in the resultant image which were originally assigned to be the phase(s) of interest (cement in this first case), a threshold operation is performed to create the appropriate volume fractions of the two phases. For example, if a cement pixel of interest has an $\mathrm{R}$-value above a critical threshold, it is reassigned to be the aluminate phase. If not, it is assigned to be the silicate phase. The critical threshold is determined such that after the threshold operation, the fraction of pixels which have been reassigned will correspond to the desired volume fraction for the reassigned phase.

After this algorithm is executed to separate the cement (non gypsum) particles into silicates and aluminates, the appropriate volume fractions of these two "phases" exist in the generated three-dimensional image. However, it remains to match the surface area fractions as well. To do this, a pixel rearrangement algorithm, based on analysis of local 3-D curvature $[21,22]$ is employed. The local curvature is simply defined to be proportional to the fraction of pixels in some local neighborhood (e.g., a $3 \times 3 \times 3$ box or sphere) which are assigned to be porosity. Here, pixels of one solid phase located at high curvature sites are exchanged with pixels of the other solid phase located at low curvature sites. This changes the fraction of each phase in contact with the pore space so that the surface area fractions of each phase can be made to match the perimeter fractions present in the original two-dimensional SEM image.

Once this phase separation is accomplished for converting the "cement" into the silicates and aluminates, the algorithms are executed on the developing 3-D image two more times. The silicates are further segmented into $C_{3} S$ and $C_{2} S$, while the aluminates are further divided into $C_{3} A$ and $C_{4} A F$. Figure 5 shows a portion of an initial generated 3-D microstructure for Cement 116 at a $w / c$ ratio of 0.4 .

\subsection{3-D Cement Hydration Model}

The cement hydration model was originally developed in two dimensions [23] to operate directly on SEM images such as those in Figs. 2 and 3. Here, the model has been extended 


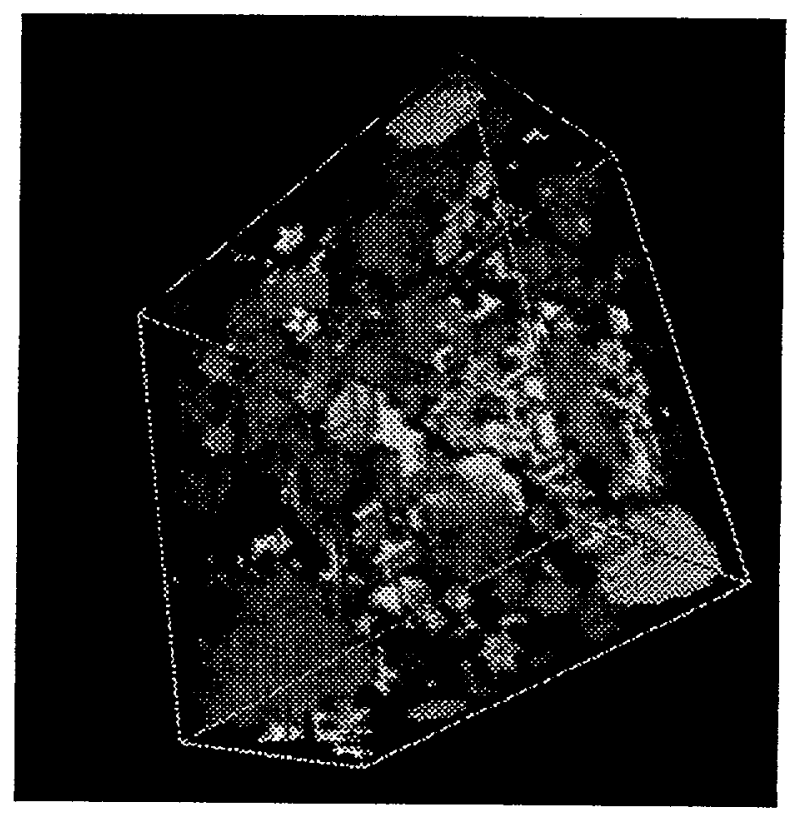

Figure 5: Portion of initial 3-D image of Cement 116 with $w / c=0.4$. Phases from brightest to darkest are: $C_{3} A$, gypsum, $C_{4} A F, C_{3} S, C_{2} S$, and porosity.

to three dimensions, additions made to determine model heat of hydration and chemical shrinkage, and several coefficients adjusted to better model the experimental data generated for actual cements. To begin, one must decide the phases and reactions to consider in the cement hydration model. Table 6 provides a list of the phases included in the present version of the three-dimensional cement hydration model, along with their densities, molar volumes, $[24,25]$ and heats of formation $[26,27]$. Figure 6 summarizes the reactions included in the current version of the model, as modified from those provided in [23]. The volume stoichiometries indicated below each reaction have been calculated based on the molar stoichiometries of the reactions and the compound molar volumes tabulated in Table 6 .

The reactions provided in Fig. 6 are implemented as a series of cellular automata-like rules which operate on the original three-dimensional representation of cement particles in water. Rules are provided for the dissolution of solid material, the diffusion of the generated diffusing species, and the reactions of diffusing species with each other and with solid phases. These rules are summarized in the state transition diagram provided in Fig. 7 . Their implementation is as follows.

For dissolution, first, an initial scan is made through all pixels (elements) present in the 3$\mathrm{D}$ microstructure, to identify all pixels which are in contact with pore space. Thus, any solid pixels which have one or more immediate ( \pm 1 in the $x, y$, or $z$ directions) neighbors which are classified as porosity are eligible for dissolution. In addition, each solid phase is characterized by two dissolution parameters, a solubility flag and a dissolution probability. The solubility flag indicates if a given phase is currently soluble during the hydration process, with a value of 1 indicating that the phase is soluble. The initial cement phases are always soluble during the hydration process. Conversely, some phases, like ettringite, are initially insoluble but become soluble during the hydration (e.g., when the gypsum is nearly consumed). The 


$$
\begin{aligned}
& \text { Silicate Reactions } \\
& \mathrm{C}_{3} \mathrm{~S}+5.3 \mathrm{H} \rightarrow \mathrm{C}_{1.7} \mathrm{SH}_{4}+1.3 \mathrm{CH} \\
& \begin{array}{llll}
1 & 1.34 & 1.521 & 0.61
\end{array} \\
& \mathrm{C}_{2} \mathrm{~S}+4.3 \mathrm{H} \rightarrow \mathrm{C}_{1.7} \mathrm{SH}_{4}+0.3 \mathrm{CH} \\
& \begin{array}{llll}
1 & 1.49 & 2.077 & 0.191
\end{array} \\
& \mathrm{C}_{3} \mathrm{~A}+6 \mathrm{H} \rightarrow \mathrm{C}_{3} \mathrm{AH}_{6} \\
& 1 \quad 1.21 \quad 1.69 \\
& \mathrm{C}_{3} A+3 C \bar{S} H_{2}+26 H \rightarrow C_{6} A \bar{S}_{3} H_{32} \\
& \begin{array}{llll}
0.4 & 1 & 2.1 & 3.3
\end{array} \\
& 2 C_{3} A+C_{6} A \bar{S}_{3} H_{32}+4 H \rightarrow 3 C_{4} A \bar{S} H_{12} \\
& \begin{array}{llll}
0.2424 & 1 & 0.098 & 1.278
\end{array} \\
& \mathrm{C}_{4} A F+3 \mathrm{C} \bar{S} \mathrm{H}_{2}+30 \mathrm{H} \rightarrow \mathrm{C}_{6} A \bar{S}_{3} \mathrm{H}_{32}+\mathrm{CH}+\mathrm{FH}_{3} \\
& \begin{array}{llllll}
0.575 & 1 & 2.426 & 3.3 & 0.15 & 0.31
\end{array} \\
& 2 C_{4} A F+C_{6} \bar{S}_{3} H_{32}+12 \mathrm{H} \rightarrow 3 C_{4} A \bar{S} H_{12}+2 \mathrm{CH}+2 \mathrm{FH}_{3} \\
& \begin{array}{llllll}
0.348 & 1 & 0.294 & 1.278 & 0.09 & 0.19
\end{array}
\end{aligned}
$$

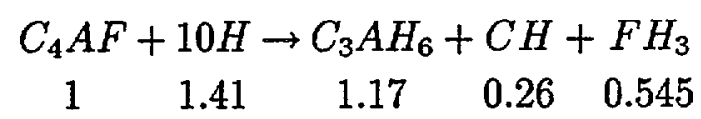

Figure 6: Cement model reactions - numbers below reactions indicate volume stoichiometries. 
Table 6: Physical Properties of Cementitious Materials

\begin{tabular}{lllll}
\hline \multicolumn{1}{c}{ Compound Name } & $\begin{array}{l}\text { Compound } \\
\text { Formula }\end{array}$ & $\begin{array}{l}\text { Density } \\
\left(\mathrm{Mg} / \mathrm{m}^{3}\right)\end{array}$ & $\begin{array}{l}\text { Molar volume } \\
\left(\mathrm{cm}^{3} / \mathrm{mole}\right)\end{array}$ & $\begin{array}{l}\text { Heat of formation } \\
(\mathrm{kJ} / \mathrm{mole})\end{array}$ \\
\hline & & & & \\
Tricalcium silicate & $C_{3} S$ & 3.21 & 71. & -2927.82 \\
Dicalcium silicate & $C_{2} S$ & 3.28 & 52. & -2311.6 \\
Tricalcium aluminate & $C_{3} A$ & 3.03 & 89.1 & -3587.8 \\
Tetracalcium aluminoferrite & $C_{4} A F$ & 3.73 & 128 & -5090.3 \\
Gypsum & $C \bar{S} H_{2}$ & 2.32 & 74.2 & -2022.6 \\
Calcium silicate hydrate, $\mathrm{C}-\mathrm{S}-\mathrm{H}$ & $\mathrm{C}_{1.7} S H_{4}$ & 2.12 & 108 & -3283. \\
Calcium hydroxide & $C H$ & 2.24 & 33.1 & -986.1 \\
Ettringite & $C_{6} A \bar{S}_{3} H_{32}$ & 1.7 & 735. & -17539. \\
Monosulfate & $C_{4} A \bar{S} H_{12}$ & 1.99 & 313. & -8778. \\
Hydrogarnet & $C_{3} A H_{6}$ & 2.52 & 150. & -5548. \\
Iron hydroxide & $F H_{3}$ & 3.0 & 69.8 & -823.9 \\
\hline
\end{tabular}

calcium hydroxide is made to be soluble to allow Ostwald ripening of the smaller calcium hydroxide crystals into larger ones. The second parameter indicates the relative probability of a phase dissolving when a pixel containing that phase "steps" into pore space. This is included in the model to allow the cement minerals to react at different rates as has been observed experimentally [24]. In the current model configuration, the $C_{3} A$ and $C_{3} S$ are assigned relatively high dissolution probabilities (>0.8) while the $C_{4} A F$ and $C_{2} S$ are given relatively low ones $(<0.2)$. Since the latter two phases generally account for less than $30 \%$ of the cement, variations in their dissolution probabilities will not have a major effect on the results of the hydration model, although recent research has shown that enhancing the dissolution of $C_{4} A F$ can significantly influence the properties of cements with substantial $C_{4} A F$ fractions [28].

In a second pass through the microstructure, all identified surface pixels are allowed to take a one step random walk. If the step lands the pixel in porosity, the phase comprising the pixel is currently soluble, and dissolution is determined to be probable (by comparing a $\mathrm{U}[0,1)$ random number to the dissolution probablility), the dissolution is allowed and one or more diffusing species are generated as indicated in Fig. 7. If the dissolution is not allowed, the surface pixel simply remains as its current solid phase, but may dissolve later in the hydration. The locations of all diffusing species are stored in a linked list data structure which can expand and contract dynamically during execution to optimize memory usage. In this way, unlike in previous versions of the NIST model [3,23], diffusing species may remain in solution from one dissolution phase to the next. Previously, all diffusing species were reacted before a new dissolution step was performed.

The generated diffusing species execute random walks in the available pore space, until they react according to the rules provided in Fig. 7. For each diffusing species, the reaction rules included in the present version of the 3-D cement hydration model are as follows:

diffusing $C-S-H$ : when a diffusing $C-S-H$ species collides with either solid $C_{3} S$ or $C_{2} S$ or previously deposited $C-S-H$, it is converted into solid $C-S-H$ with a probability of 1 . 


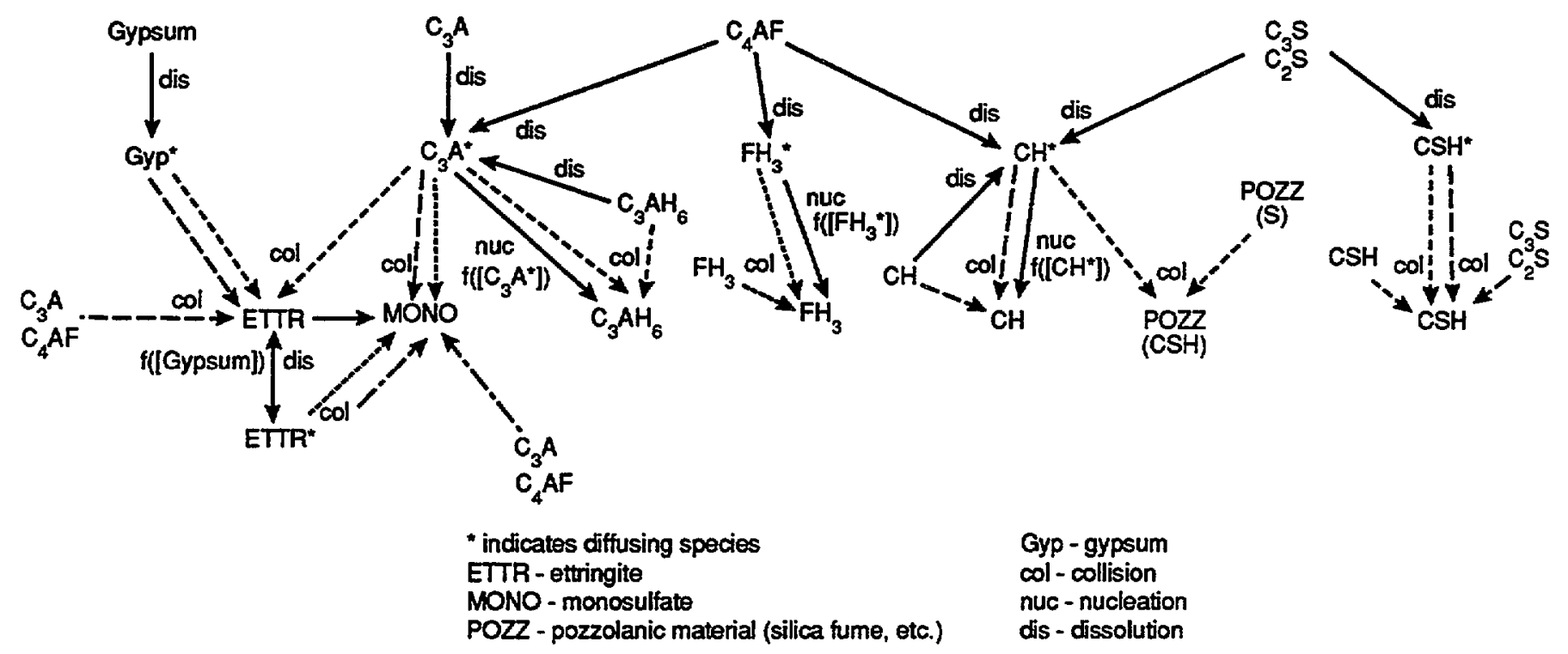

Figure 7: State transition diagram for 3-D Cement Hydration Model. Arrow patterns denote the collision of two species to form a hydration product. $\mathrm{f}([\mathrm{X}])$ denotes that nucleation or dissolution probability is a function of concentration or volume fraction of phase $\mathrm{X}$.

diffusing $\mathrm{CH}$ : for each diffusion step, a random number is generated to determine if nucleation of a new $C H$ crystal is probable; if so, the diffusing $C H$ is converted into solid $C H$ at its present location. In addition, if a diffusing $C H$ collides with solid $C H$, it is converted into solid $C H$ with a probability of 1 .

diffusing $\mathrm{FH}_{3}$ : for each diffusion step, a random number is generated to determine if nucleation of a new $\mathrm{FH}_{3}$ crystal is probable; if so, the diffusing $\mathrm{FH}_{3}$ is converted into solid $\mathrm{FH}_{3}$ at its present location. In addition, if a diffusing $\mathrm{FH}_{3}$ collides with solid $\mathrm{FH}_{3}$, it is converted into solid $\mathrm{FH}_{3}$ with a probability of 1 .

diffusing gypsum: the diffusing gypsum can only react by collision with some other species in the microstructure. If it collides with solid $C-S-H$, it can be absorbed as long as the previously absorbed gypsum is less than some constant (e.g., 0.01 ) multiplied by the number of solid $C-S-H$ pixels currently present in the system. If it collides with either solid or diffusing $C_{3} A$, ettringite is formed. If it collides with solid $C_{4} A F$, ettringite, $\mathrm{CH}$, and $\mathrm{FH}_{3}$ are formed to maintain the appropriate volume stoichiometry as shown in Fig. 6.

diffusing ettringite: when diffusing ettringite is created, it also reacts only by collision with other species. If it collides with solid or diffusing $C_{3} A$, monosulfoaluminate is formed. If it collides with solid $C_{4} A F$, monosulfoaluminate, $C H$, and $F H_{3}$ are formed. Finally, if it collides with solid ettringite, there is a small probability that it is converted back into solid ettringite. This latter rule is provided to avoid the possibility of a large buildup of diffusing ettringite in the microstructure.

diffusing $C_{3} A$ : If nucleation is probable or the diffusing $C_{3} A$ collides with solid $C_{3} A H_{6}$ and precipitation is probable, solid $C_{3} A H_{6}$ is formed. If it collides with diffusing gypsum, ettringite is formed. If it collides with diffusing or solid ettringite, monosulfoaluminate is formed. 
For $\mathrm{C}_{3} A H_{6}, \mathrm{CH}$, and $\mathrm{FH}_{3}$, the probability of nucleation, $P_{n u c}$, of diffusing species is governed by an equation of the form:

$$
P_{n u c}\left(C_{i}\right)=A_{i} *\left(1-e^{\frac{-\left[C_{j}\right]}{\left.T B_{i}\right]}}\right)
$$

where $C_{i}$ is the current number of diffusing species $i$ and $A_{i}$ and $B_{i}$ are constants which control the number and rate at which crystals are formed in the microstructure. This results in the effect that few new crystals are formed late in the hydration when the "concentrations" of diffusing species are reduced relative to their initial values, in agreement with experimental observations [29].

In general, the hydration reaction products are allowed to grow with a completely random morphology. An exception to this is ettringite, where an attempt is made to grow the solid ettringite as needle-like structures by evaluating the surface curvature using a pixel counting algorithm [21, 22]. When new ettringite is forming, an attempt is made to maximize the number of non-ettringite pixels in contact with the new ettringite pixel. This will naturally result in the formation of maximum surface area (or needle-like) ettringite structures.

Prior to each dissolution, the 3-D microstructure is scanned to determine the number of pixels of each phase currently present in the system. From these volumes, chemical shrinkage and heat of hydration can be calculated. The chemical shrinkage is calculated by determining the amount of water consumed by reaction (based on the values in Table 6) in comparison to the volume of capillary porosity remaining in the microstructure. For low $w / c$ ratio systems, all of the water may be consumed while some capillary porosity remains. However, in the execution of the model, it is assumed that saturation is always maintained to simulate the experimental measurements performed in this study, where additional water is always present on the top surface of the hydrating cement paste. Research to extend the model to hydration under sealed conditions, with the creation of internal voids due to chemical shrinkage, is ongoing. The heat of hydration can be based on the heats of formation given in Table 6, or the tabulated enthalpy values for each of the four major phases as listed in Table 7. For the model, degree of hydration is calculated as the mass of cementitious material which has reacted divided by the starting mass of cement.

Table 7: Enthalpy of Complete Hydration for Major Phases of Cement

\begin{tabular}{ccc}
\hline Phase & Enthalpy (kJ/kg phase) & Source \\
\hline & & \\
$C_{3} S$ & 517 & {$[8]^{a}$} \\
$C_{2} S$ & 262 & {$[8]$} \\
$C_{3} A$ & 1144 & {$[8]$} \\
$C_{4} A F$ & 725 & {$[26]^{b}$} \\
\hline
\end{tabular}

${ }^{a} w / c=0.4$ and $\mathrm{T}=21^{\circ} \mathrm{C}$

${ }^{b} w / c=0.5$ and $\mathrm{T}=20^{\circ} \mathrm{C}$

\subsection{Comments on Assumptions of the Hydration Model}

As with any model, the results of the 3-D cement hydration model are dependent on the underlying assumptions that have been made in creating it. Because of the current state of 
knowledge of cementitious systems, numerous assumptions had to be incorporated into the current version of the model. While an effort has been made to include as many realistic features into the model as possible, only time will tell if the underlying assumptions are indeed reasonable. However, the usefulness of a model may be somewhat independent of the validity of the underlying assumptions. If a model, even one formulated on what are subsequently proven to be invalid or partially valid assumptions, leads to new fruitful avenues of research or provides accurate predictive capabilities, it has served a valuable purpose.

While not exhaustive, the following is an attempt to list the major assumptions underlying the current version of the NIST microstructure model. First, as mentioned previously, we are assuming digitized spherical shapes for all of the cement particles. While the particles in Figs. 2 and 3 definitely exhibit somewhat elongated shapes, spheres appear to be a reasonable simplification. From a computational standpoint, the generation of ellipsoidal shapes or even randomly oriented non-uniform particles would be straightforward. To do this, however, some measures of the three-dimensional nature of the cement particles would have to be derived from the two-dimensional images or perhaps directly assessed on threedimensional $x$-ray microtomographic images [30]. A further assumption of the initial 3-D cement particle image generation is that the PSD for the gypsum is the same as that for the ground cement clinker. Here, if the PSD of the gypsum had been assessed separately in the case of added, as opposed to interground, gypsum, that information could be directly included in the 3-D generation algorithms.

Concerning the phases present in the cement clinker, currently, the sodium and potassium sulfate are not accounted for in the model. These alkali sulfates are known to effect the early reactivity of cements [8]. The imaging techniques described previously could be extended by acquiring $\mathrm{x}$-ray images for $\mathrm{Na}$ and $\mathrm{K}$. Then, it would be necessary to hypothesize the reactions in which the alkali ions $\left(\mathrm{Na}^{+}\right.$and $\left.\mathrm{K}^{+}\right)$participate and the amount of substitution of these ions for $\mathrm{Ca}^{++}$, etc. in the already-considered hydration products. Due to these and other complications, the current version of the hydration model focuses on the postinduction period of the hydration of portland cement, as our major interest lies in the long term properties of cement-based materials.

Concerning the cement hydration model itself, all diffusing species "randomly diffuse" at the same rate in the available pore space. However, a higher mobility for calcium, aluminate, and sulfate ions is implied by the rule that, upon dissolution, diffusing $C_{3} A$, diffusing $\mathrm{CH}$, and diffusing gypsum are located at totally random locations in the available pore space. This results in a somewhat uniform distribution of these species in the pore space. Conversely, diffusing $\mathrm{FH}_{3}$, diffusing $\mathrm{C}-\mathrm{S}-\mathrm{H}$, and diffusing ettringite are located at or near the dissolution source, implying a lower mobility for the iron and silicate ionic species and leading to localized concentration profiles. Additionally, no explicit relationships to ion concentrations and solubility products are considered during model execution. (It should be noted that recent efforts by other research groups have concentrated on developing reactiondiffusion models for cement hydration which explicitly account for solubility products and diffusion coefficients for the relevant species [31]). As mentioned previously, the dissolution probability of ettringite is biased to avoid a buildup of diffusing ettringite species in the pore space. Similarly, the dissolution probabilities of $C H$ and $C_{3} A H_{6}$ are also adjusted based on the current "concentrations" of diffusing species. Further assumptions are the form of the equation for the nucleation probabilities provided in Equation 5 and the provision for $C_{3} A H_{6}$, ettringite, and monosulfoaluminate to co-exist. Although, in the latter case, the $C_{3} A H_{6}$ and ettringite should lead to the formation of more monosulfoaluminate, the kinetics 
of this reaction could be quite slow in a hydrated cement paste system, leading to local regions where one of the three phases is dominant, such that all three phases would be detected in a hydrated cement paste [8]. As further experimental and theoretical data becomes available, the model can be adapted to better represent the physical reality since the overall framework of a cellular-automata-based model is inherently flexible [23].

\section{Results and Discussion}

\subsection{Experimental Results}

Figure 8 provides a plot of the normalized experimental results for Cements 115 and 116 for $w / c$ ratios of $0.3,0.4$, and 0.45 . In this figure, the heat of hydration values have been normalized by the values calculated based on the Bogue potential phase compositions of the cements and the tabulated heats of hydration of the major phases provided in Table 7. The non-evaporable water contents have been normalized by the values measured experimentally on the high $w / c$ mixes as described in the experimental section. Finally, the chemical shrinkage values have been normalized by the value (within $\pm 0.01 \mathrm{~mL} / \mathrm{g}$ cement) which gives the best fit to the non-evaporable water content data for the $w / c=0.45$ systems. The $w / c=0.45$ data were chosen because these results are not affected by the depercolation of the capillary porosity, as will be discussed below. This value was then held constant at the lower $w / c$ ratios. As can be seen in Figure 8, excellent agreement is observed between the three measured properties (non-evaporable water content, heat release, and chemical shrinkage). A previous study by Parrott et al. [32] has produced similar agreement, finding "a directly proportional relationship between the heat of hydration and chemical shrinkage." Geiker [11] has noted a linear relationship between chemical shrinkage and non-evaporable water content for an ordinary portland cement with $w / c=0.5$, cured at $20^{\circ} \mathrm{C}$. In addition, in 1935, Powers [33] reported a linear relationship between heat of hydration and water absorbed during hydration for four different cements, with a constant of proportionality of 19.3 (cal/g)/(g water/100 g cement). For the results in Fig. 8, we find values of 16.9 and 20.6 for Cements 115 and 116 respectively, in good agreement with Powers' results.

One interesting observation can be made concerning the chemical shrinkage data for the lower $w / c$ ratios in Fig. 8. For both the 0.3 and $0.4 w / c$ ratios, one can observe that at longer times, the chemical shrinkage curves diverge away from the non-evaporable water content data. In every case, the chemical shrinkage is seen to lie below the non-evaporable water data at these long times. As has been suggested by Geiker [11], this is due to the depercolation of the capillary porosity in the hydrating cement paste. As hydration occurs, depending on the initial $w / c$ ratio, a point will be reached where the capillary porosity is no longer connected, and transport must then occur through the much smaller gel pores in the $C-S-H$ gel [4]. Since this transport will occur at a much slower rate, the rate at which water is absorbed into the specimen will fall below the rate at which empty voids are being generated, leading to the observed divergence in the experimental curves. The horizontal lines provided in Fig. 8 indicate the degree of hydration needed to achieve this capillary pore discontinuity, based on the results of the original $C_{3} S$ hydration model $[3,4]$. The agreement between the experimental observations and the predicted point of discontinuity is quite good, particularly for the $w / c=0.3$ data sets. This pore discontinuity has also recently been observed using impedance spectroscopy measurements on partially frozen cement paste specimens [34]. 

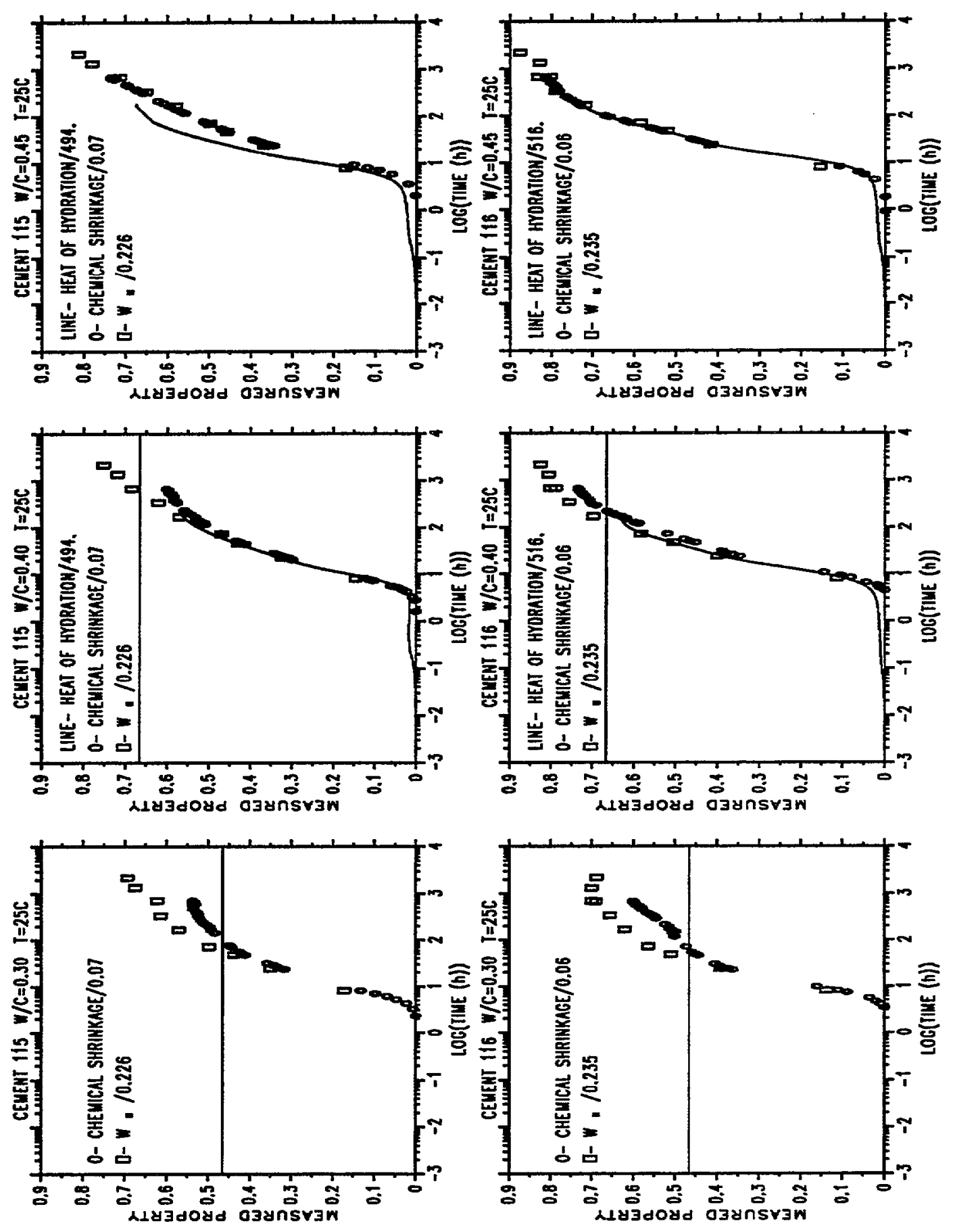

Figure 8: Experimental results for CCRL Cements and 115 and 116 vs. time. 
The heats of hydration measured after seven days using the NIST microcalorimeter can be compared to the values determined using the heat of solution technique in the CCRL proficiency sample program [5]. Table 8 summarizes the results for Cements 115 and 116. The values measured using the microcalorimeter are below, but within 1.3 standard deviations, of the CCRL reported values. These lower values would be expected since the results here do not include the contribution of the initial exothermic mixing peak, as discussed in the experimental section. Additionally, the mixing of the samples for the standard heat of solution method [6] may be more complete than that achieved in the small microcalorimeter cells, also contributing to a greater heat release in the former case. However, it is encouraging to note that the differences in heat release between Cements 115 and 116 are basically identical using the two techniques, being 49 and $50 \mathrm{~kJ} / \mathrm{kg}$ for the mean heat of solution and microcalorimeter measurements, respectively.

Table 8: Measured Seven Day Heats of Hydration for CCRL Cements 115 and 116

\begin{tabular}{cccc}
\hline Cement & $\begin{array}{c}\text { Heat of Solution } \\
\text { Method }(\mathrm{kJ} / \mathrm{kg})[5]\end{array}$ & $\begin{array}{c}\text { Heat of Solution } \\
\text { Standard Deviation }(\mathrm{kJ} / \mathrm{kg})[5]\end{array}$ & $\begin{array}{c}\text { NIST microcalorimeter } \\
\text { Method }(\mathrm{kJ} / \mathrm{kg})\end{array}$ \\
\hline & & & \\
115 & 311 & 28 & 277 \\
116 & 360 & 26 & 327 \\
\hline
\end{tabular}

To calibrate the model to the experimental results, both are fitted to the same functional form. In the literature, a variety of models have been used to fit either degree of hydration or strength development vs. time [35], mainly in connection with the application of the maturity method to concrete strength development. Two commonly used models are the linear and parabolic dispersion models originally developed by Knudsen [36]. The linear model for a property of interest, $A$, is as follows:

$$
A=A_{u} \frac{k\left(t-t_{0}\right)}{1+k\left(t-t_{0}\right)}
$$

where $A_{u}$ is the ultimate achievable value of the property, $t_{0}$ is an induction time, and $k$ is a rate constant. The parabolic model is similar, taking the form:

$$
A=A_{u} \frac{k \sqrt{\left(t-t_{0}\right)}}{1+k \sqrt{\left(t-t_{0}\right)}} .
$$

Depending on the particular cement being studied, Geiker has noted that one of the two above equations will generally provide the better fit to experimental data [11]. Thus, both equations were fitted to the experimental data for non-evaporable water content vs. time. The non-evaporable water content data was selected (as opposed to heat of hydration or chemical shrinkage), because experimental values were collected for periods of up to 90 days.

Equations 6 and 7 were fitted to the experimental data using non-linear regression analysis available in DATAPLOT [37], a graphical analysis software package developed at NIST. Figure 9 provides a representative example of the fit of the two equations to the experimental data; all of the graphs for the two cements and three $w / c$ ratios are provided in Appendix 
A. Tables 9 and 10 summarize the results of the regression analysis, including the residual standard deviations of the fits. In general, for Cements 115 and 116, the better fit to the experimental non-evaporable water content data was provided by Equation 7, as indicated by the lower residual standard deviation. In addition, using this model, the values of $t_{0}$ are relatively constant for the three $w / c$ ratios for each of the two cements and the values of $A_{u}$ for the $w / c=0.45$ data sets are fairly close to the values of 0.226 and 0.235 measured on the high $w / c$ ratio pastes of 115 and 116 respectively, as would be expected for $w / c$ values greater than about $0.42[8,25]$. Based on these considerations, this equation will also be used to fit the model results, for calibration against experiment.
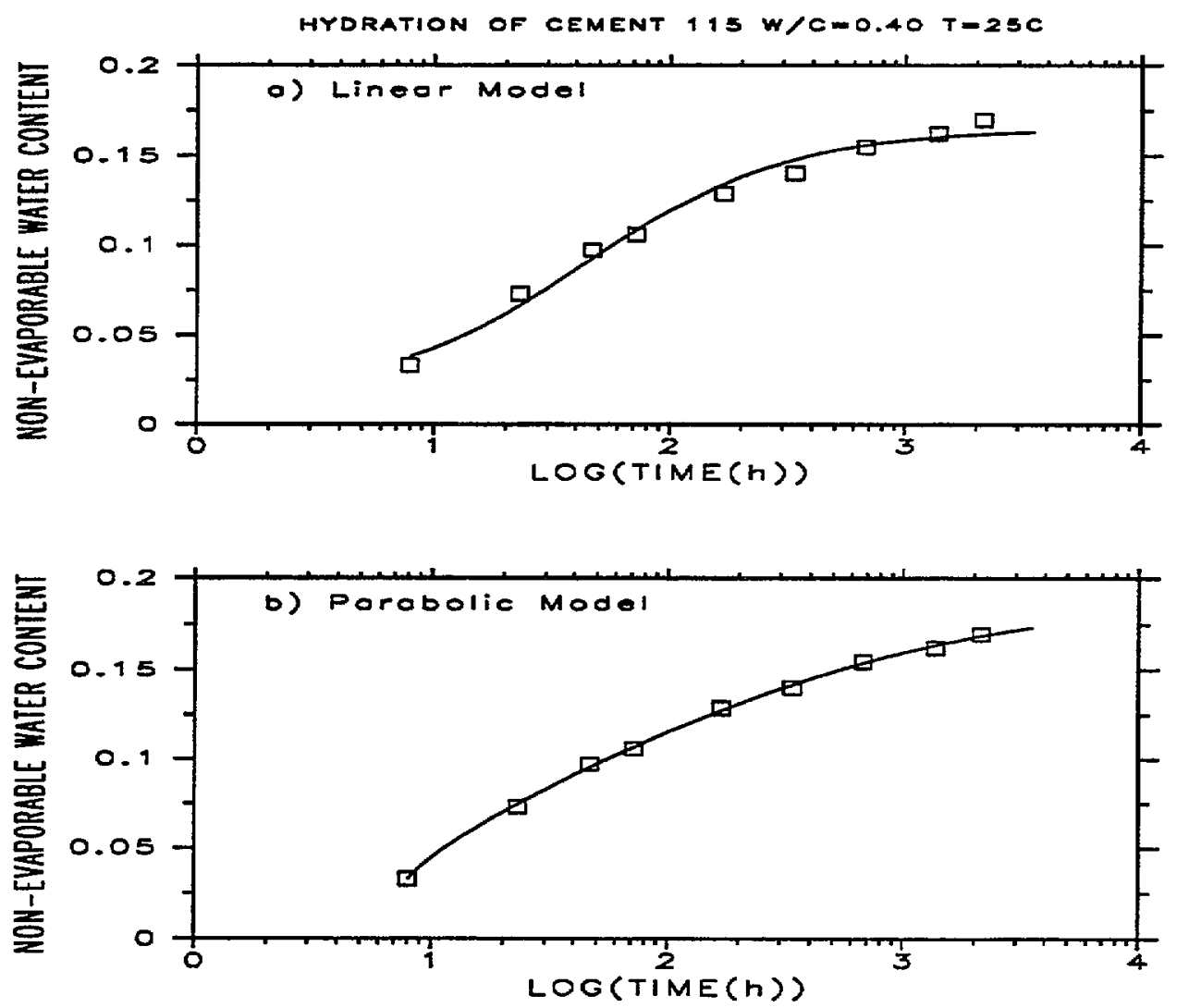

Figure 9: Fits of Knudsen's dispersion models to experimental non-evaporable water content (g $\mathrm{H}_{2} \mathrm{O} / \mathrm{g}$ cement) vs. time.

\subsection{Model Results}

Some general results of the microstructure model will first be presented, before proceeding to the calibration of model hydration rate, heat release, and chemical shrinkage against the experimental data. Results can be conveniently summarized by plotting the phase volume fraction vs. number of elapsed dissolution cycles for each phase present in the model. Typical results are illustrated in Figures 10 through 12 which provide a series of graphs for Cement 116 at $w / c=0.4$. For the anhydrous phases $\left(C_{3} S\right.$, etc. $)$, the phase fractions are seen to monotonically decrease with cycles, but at rates proportional to the assigned dissolution probabilities of the phase (i.e., $C_{3} S$ and $C_{3} A$ react at higher rates than $C_{2} S$ and $C_{4} A F$ ). 
Table 9: Parameters for Knudsen's Linear Dispersion Model for Cements 115 and 116

\begin{tabular}{cllccc}
\hline Cement & $w / c$ & $A_{u}\left(\mathrm{~g} \mathrm{H}_{2} \mathrm{O} / \mathrm{g}\right.$ cement $)$ & $k\left(\mathrm{~h}^{-1}\right)$ & $t_{0}(\mathrm{~h})$ & Res. Std. Dev. \\
\hline & & & & & \\
115 & 0.3 & 0.152 & 0.0382 & -1.52 & 0.0044 \\
115 & 0.4 & 0.165 & 0.0254 & -3.55 & 0.0064 \\
115 & 0.45 & 0.176 & 0.0219 & -8.02 & 0.0086 \\
116 & 0.3 & 0.164 & 0.0607 & 3.93 & 0.0026 \\
116 & 0.4 & 0.193 & 0.0385 & 3.39 & 0.0043 \\
116 & 0.45 & 0.201 & 0.0338 & 0.77 & 0.0055 \\
\hline
\end{tabular}

Table 10: Parameters for Knudsen's Parabolic Dispersion Model for Cements 115 and 116

\begin{tabular}{clllcc}
\hline Cement & $w / c$ & $A_{u}\left(\mathrm{~g} \mathrm{H}_{2} \mathrm{O} / \mathrm{g}\right.$ cement) & $k\left(h^{-1 / 2}\right)$ & $t_{0}(\mathrm{~h})$ & Res. Std. Dev. \\
\hline & & & & & \\
115 & 0.3 & 0.171 & 0.218 & 6.23 & 0.0033 \\
115 & 0.4 & 0.193 & 0.154 & 6.22 & 0.0015 \\
115 & 0.45 & 0.207 & 0.145 & 5.42 & 0.0029 \\
116 & 0.3 & 0.181 & 0.299 & 7.49 & 0.0048 \\
116 & 0.4 & 0.221 & 0.197 & 7.54 & 0.0048 \\
116 & 0.45 & 0.231 & 0.187 & 7.04 & 0.0052 \\
\hline
\end{tabular}

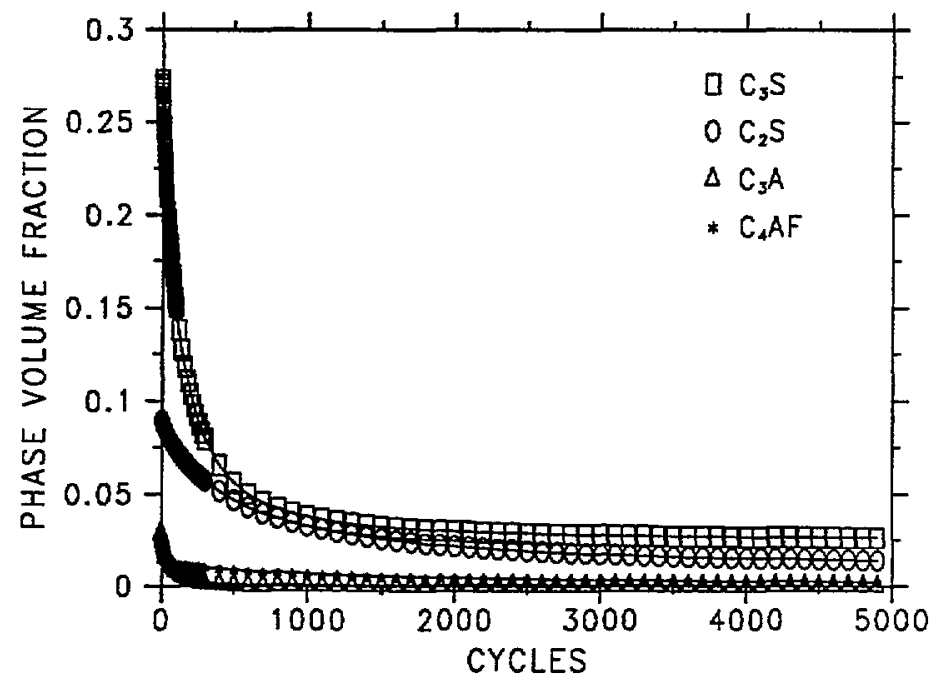

Figure 10: Model anhydrous cement volume fractions vs. elapsed cycles for Cement 116 with $w / c=0.4$. 
Similarly, as shown in Fig. 11, porosity decreases monotonically with cycles. $\mathrm{C}-\mathrm{S}-\mathrm{H}, \mathrm{CH}$ and $\mathrm{FH}_{3}$ all are seen to increase monotonically with cycles.

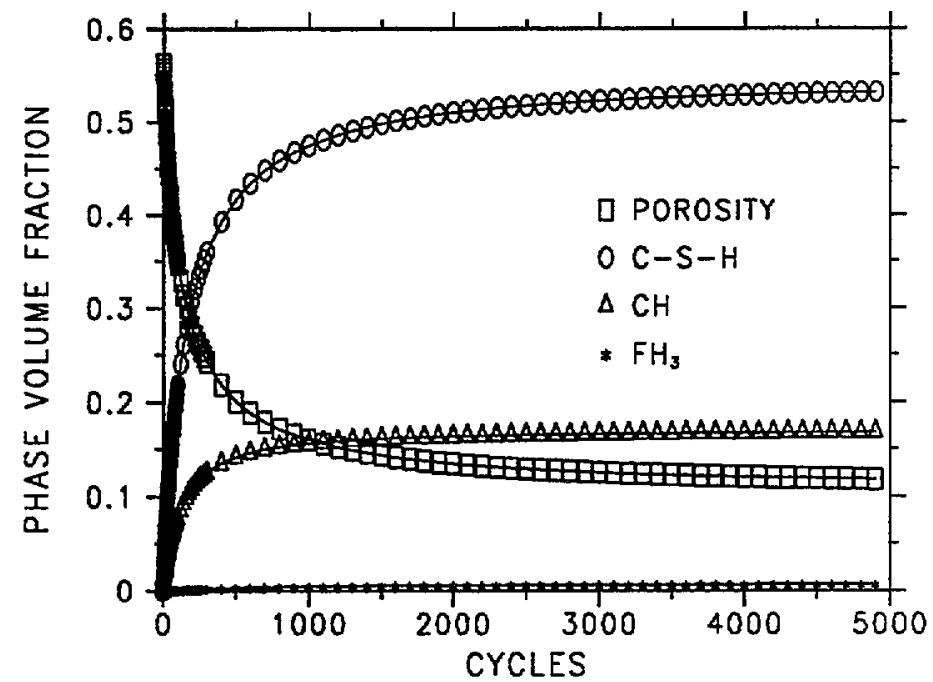

Figure 11: Model porosity and reaction product volume fractions vs. elapsed cycles for Cement 116 with $w / c=0.4$.

The behavior of the aluminate hydration products is more complex as shown in Fig. 12. Here, while gypsum remains in the system at a significant level ( $>10 \%$ of its initial volume), mostly ettringite (and a little $C_{3} A H_{6}$ ) is formed from the reaction of the aluminate phases with gypsum. When the gypsum is nearly consumed, the formation of the monosulfoaluminate phase (Afm) begins and the supply of ettringite is gradually depleted, while more $C_{3} A H_{6}$ continues to form. The initiation of monosulfoaluminate formation prior to the complete depletion of gypsum is consistent with recent experimental results [38]. The shapes of the curves for the ettringite buildup and decay and the Afm buildup are quite similar to those found in the literature [28], as measured using X-ray diffraction on pastes in which the dissolution of the ferrite phase had been specially activated. In Fig. 12, the ettringite peaks to a maximum volume fraction at about $60-70$ cycles. Later results will present the calibration of model cycles against experimental time; such results indicate that 60-70 cycles corresponds to about $12-15$ hours of real time for these cements. This is a reasonable time for the conversion of ettringite to monosulfoaluminate to begin, as indicated by a secondary peak in calorimetry measurements $[8,39]$. Such a shoulder (peak) on the heat release curve can be clearly observed for the heat release signal curve for Cement 116 in Fig. 4 (occurring at about 750 minutes). However, some researchers [40] have suggested that this secondary heat peak is associated with the renewed formation of ettringite and not the conversion of ettringite to monosulfoaluminate. It should be recognized that model parameters could be adjusted to obtain this depletion of gypsum at any specific time. Here, the relative agreement with conventional experimental observations is rather fortuitous, as no specific attempt was made to achieve this gypsum depletion at a specific time. Rather, the relative dissolution probabilities of the phases were set a priori at reasonable values based on data in the literature [8].

Concerning the model heat release data, the values calculated based on the major phase enthalpies at complete hydration can be compared to those based on the tabulated heats 


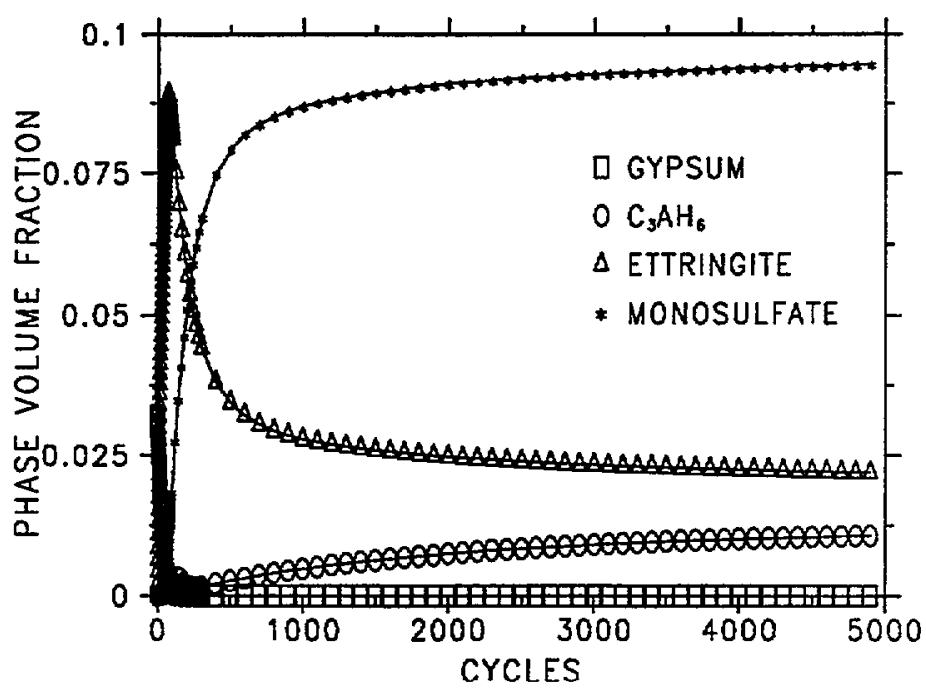

Figure 12: Model aluminate reaction product volume fractions vs. elapsed cycles for Cement 116 with $w / c=0.4$.

of formation of all of the phases. Figure 13 provides a comparison of these two results for Cement 116 with $w / c=0.45$. The two curves are seen to overlap early in the hydration, but diverge in the later stages. This is partially an artifact of the diffusing species remaining in solution from one dissolution cycle to the next in the model, as heats of formation can be only approximately applied to these species since multiple reaction paths are possible. Late in the hydration, a small but significant quantity of diffusing ettringite and diffusing $C_{3} A$ species are observed to build up in the pore space. Conversely, when using the major phase enthalpies at complete hydration, the heat release can be updated completely after each dissolution cycle, based on the amount of remaining unhydrated cement phases. Since the experimentally measured heats of hydration after 7 days are on the order of $300 \mathrm{~kJ} / \mathrm{kg}$, it is somewhat difficult to say which of the two model curves in Fig. 13 best represents the experimental data. However, based on the differences in heats of hydration after 7 and 28 days measured using the heat of solution technique [5], the model values based on the major phase enthalpies do provide a better agreement with the experimental data.

\subsection{Calibration of Model Using Experimental Results}

To fit the model results to those measured experimentally, a conversion between cycles and time is necessary. The simplest conversion would be to use a linear proportionality (time $=B *$ cycles). However, it has been previously pointed out to the author that the NIST cement hydration model generates kinetics which closely follow Knudsen's linear dispersion model, but not the parabolic one [41]. With this in mind, an alternative relationship between time and cycles was investigated, mainly

$$
\operatorname{time}(h)=B * \text { cycles }^{2} .
$$

In this way, the linear kinetics obeyed by the model can be adapted to the parabolic kinetics exhibited by the real cements.

To calibrate the model to the experimental results based on the non-evaporable water content data, the model results for degree of hydration were regressed in Equation 7 using 


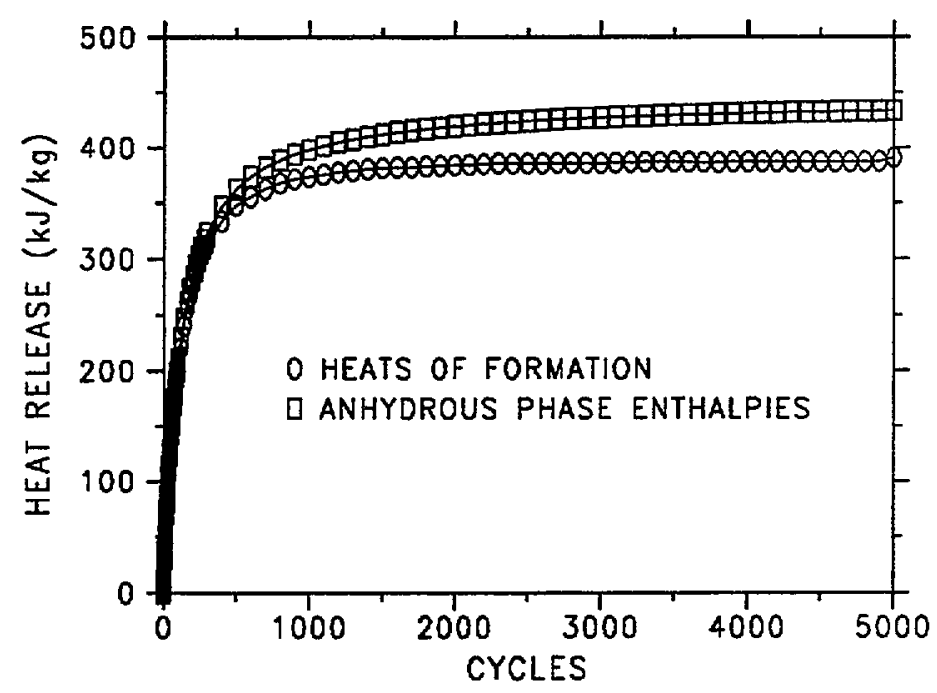

Figure 13: Comparison of heat release via major phase enthalpies and heats of formation for Cement 116 with $w / c=0.45$.

the earlier deduced parameters for $A_{u}$ and $k$, and a subset of the model degree of hydration data. This subset was generated by selecting single data points at approximately 0.05 degree of hydration intervals for values of degree of hydration between 0.10 and the amount of hydration achieved experimentally at 90 days. In this way, the regression being applied to the model is being weighted in approximately the same manner as that which was applied to the experimental results. This step was deemed necessary due to the fact that the model degree of hydration values are not evenly distributed with number of cycles (i.e., more hydration occurs during the early cycles than during the later ones). The previously determined value of $A_{u}$ was converted to degree of hydration, via normalization by the value for the nonevaporable water content at complete hydration $(0.226$ or 0.235$)$. The induction time was not included in the regression, as the NIST microstructure model makes no effort to model the induction period, but only the subsequent stages of cement hydration. Thus, this induction time will be included directly in the final equation for conversion between cycles and time, which will take the form:

$$
\operatorname{time}(h)=t_{0}+B * \text { cycles }^{2} .
$$

The determined coefficients for $\mathrm{B}$ as a function of cement and $w / c$ ratio are summarized in Table 11. Interestingly, the values for $B$ are relatively constant, suggesting that a constant value of $B$ (such as the average $B$ value of 0.00172 ) may serve to model all of the results for the two cements and three $w / c$ ratios. From the variability in results in Table 10, an average value of $t_{0}$ (namely 6.657 hours) may also suffice for these two particular cements. This value is slightly larger than the final times of set measured for the two cements using the Vicat and Gillmore needle techniques which are both on the order of 5 hours [5].

After using the non-evaporable water contents in a preliminary calibration of the model kinetics, the model chemical shrinkage results were used to determine an appropriate density for the $C-S-H$ gel. According to values in the literature [25], no chemical shrinkage would occur during the hydration of $C_{3} S$, as the volume of the products is equivalent to that of the reactants. Since the use of the literature density value led to poor predictions of chemical shrinkage for Cements 115 and 116, the molar volume of $C-S-H$ was reduced from 120 to $108 \mathrm{~cm}^{3} /$ mole. For both $C_{3} S$ and $C_{2} S$ hydration, this results in chemical shrinkages of 
Table 11: Parameter for Converting Cycles to Time for Cements 115 and 116

\begin{tabular}{lll}
\hline Cement & $w / c$ & \multicolumn{1}{c}{$B$} \\
\hline & & \\
115 & 0.3 & 0.00139 \\
115 & 0.4 & 0.00234 \\
115 & 0.45 & 0.00195 \\
116 & 0.3 & 0.00134 \\
116 & 0.4 & 0.00163 \\
116 & 0.45 & 0.00164 \\
\hline
\end{tabular}

about 6.7 (g $\mathrm{H}_{2} \mathrm{O} / \mathrm{g}$ cement) at complete hydration. For $\mathrm{C}_{3} S$ hydration, Powers [33] has directly measured a chemical shrinkage of about 5.3 ( $\mathrm{g} \mathrm{H}_{2} \mathrm{O} / \mathrm{g} \mathrm{C}_{3} \mathrm{~S}$ ) after 28 days hydration, in reasonable agreement with the value being used here. For $C_{2} S$, Powers measured a value of about $1.2\left(\mathrm{~g} \mathrm{H}_{2} \mathrm{O} / \mathrm{g} \mathrm{C}_{2} \mathrm{~S}\right)$ [33], but the $\mathrm{C}_{2} S$ would be hydrating at a slower rate than the $C_{3} S$.

Once a value(s) of $B$ has been determined and the density of $C-S-H$ specified, plots comparing model and experimental results can be generated. Appendix $B$ provides plots of the results for each of the two cements for each of the three $w / c$ ratios for hydration rate, heat release (for $w / c=0.4$ and 0.45 ), and chemical shrinkage. In these figures, the solid lines indicate the model data obtained using the specific values of $t_{0}$ and $B$ for each $w / c$ ratio and cement as given in Tables 10 and 11, while the dotted lines indicate the results that would be obtained using single average values for these parameters regardless of $w / c$ ratio and cement ID. As can be seen, the agreement between the solid lines and the experimental data is in general excellent. For the dotted lines, the agreement is similar, suggesting that for these two cements, a single relationship can be used to convert model cycles into real time. This suggests that, by capturing the particle size distribution and phase distributions of the cements, much of the hydration kinetics behavior is implicitly included in the hydration model. Knudsen [36] has previously stated that "findings by us have proven the particle size distribution to be a dominant factor in the correct modelling of cement hydration." Pommersheim [42] has also found the particle size distribution to "critically affect the kinetics" of hydration. In the following section, an attempt will be made to indicate that, in addition to particle size characterization, phase distribution quantification is important for the modelling of cement hydration.

\subsection{Discussion}

The previous results indicate that quantitative characterization of the initial cement powder can lead to the successful prediction of a variety of performance-related phenomena associated with cement hydration such as heat release and chemical shrinkage. However, at present, few laboratories have the capability of performing the SEM/X-ray analysis presented in the experimental section. With this in mind, further modelling was executed based solely on the measured particle size distributions of the cements and the Bogue-calculated potential phase compositions. For both cements being studied, starting microstructures with monophase cement particles following the measured PSD and Bogue phase fractions were created for $w / c=0.40$. Thus, for each particle placed in the three-dimensional volume, 
the phase assigned to the particle was selected based on the relative volume fractions of the phases. The same cement hydration and microstructure program was then executed to model hydration behavior and the regression analysis performed to relate model cycles to real time as described previously.

The results for these monophase particle cements are provided at the end of Appendix B. While the model follows the experimental data at early times, significant divergence occurs at longer times (seen most clearly on the degree of hydration plots). In addition, for Cement 116 , the model heat release and chemical shrinkage curves are seen to differ significantly from their experimental counterparts, more so than when multi-phase cement particles are used in the model (compare Figs. 42 and 43 to Figs. 33 and 34 ). One would expect the actual distribution of phases in the cement particles to have a greater influence at longer times, as depending on the distribution, certain phases may become totally surrounded by hydration products and be unavailable for further hydration. Recognizing that only two cements have been explored, while cement particle size distribution is certainly critical to the observed hydration kinetics, actual phase volume fractions and spatial distributions within particles also appear to be important for accurately modelling the performance of real cements at times exceeding several days.

In terms of performance variables, one key property is the compressive strength. In this study, we have also attempted to predict the compressive strength development of standard ASTM C109 [6] mortars cubes, making use of the gel-space ratio concept of Powers and Brownyard. The gel-space ratio is defined by [24]:

$$
X=\frac{0.68 \alpha}{0.32 \alpha+(w / c)}
$$

It has been shown that the compressive strength of ASTM C109 mortar cubes, $\sigma_{c}$, can be related to this gel-space ratio in the following manner [24]:

$$
\sigma_{c}=A X^{n}
$$

where $A$ represents the intrinsic strength of the cement and $n$ takes on values between 2.6 and 3.0, depending on the cement being investigated. Powers and Brownyard observed the value of $A$ to be lower for cements with higher Bogue potential $C_{3} A$ contents (e.g., $>7 \%$ ). Recently, Radjy and Vunic [43] have shown that the gel-space ratio can be employed to predict the compressive strength development of concrete based on measuring the adiabatic heat signature to estimate the degree of hydration.

Based on ASTM C109 [6], test mortars are prepared with $w / c=0.485$ for portland cement materials. Thus, model cements with $w / c=0.485$ were generated for Cements 115 and 116 using the previously described computational techniques. Since no experimental non-evaporable water content data were available, the values of $t_{0}$ and $B$ determined for each of the two cements at $w / c=0.45$ were used to convert model cycles to time based on Equation 9. From the CCRL test program, compressive strengths at 3, 7, and 28 days were available. The cement hydration and microstructure program was utilized to compute the expected degree of hydration, $\alpha$, for these cements at 3,7 , and 28 days, so that $X$ could be computed according to Equation 10. The 3-day measured compressive strength was then used to determine the value of $A$ in Equation 11, assuming an exponent $\mathrm{n}$ of 2.6. Values of $A$ of 129 and $99 \mathrm{MPa}$ were thus determined for Cements 115 and 116 , respectively. As noted above, Cement 116, with the higher $C_{3} A$ content, is observed to have the lower intrinsic strength. 
Once $A$ was determined, the model could be used to predict $\sigma_{c}$ at 7 and 28 days for comparison to the experimental data. Figures 14 and 15 present the predicted strength developments in comparison to those measured in the CCRL proficiency sample program. The standard deviation in the measured values is also included in the plots for reference purposes. The predictive ability of the model is again demonstrated, as it appears that compressive strength can be predicted well within the standard deviation of an interlaboratory test program.

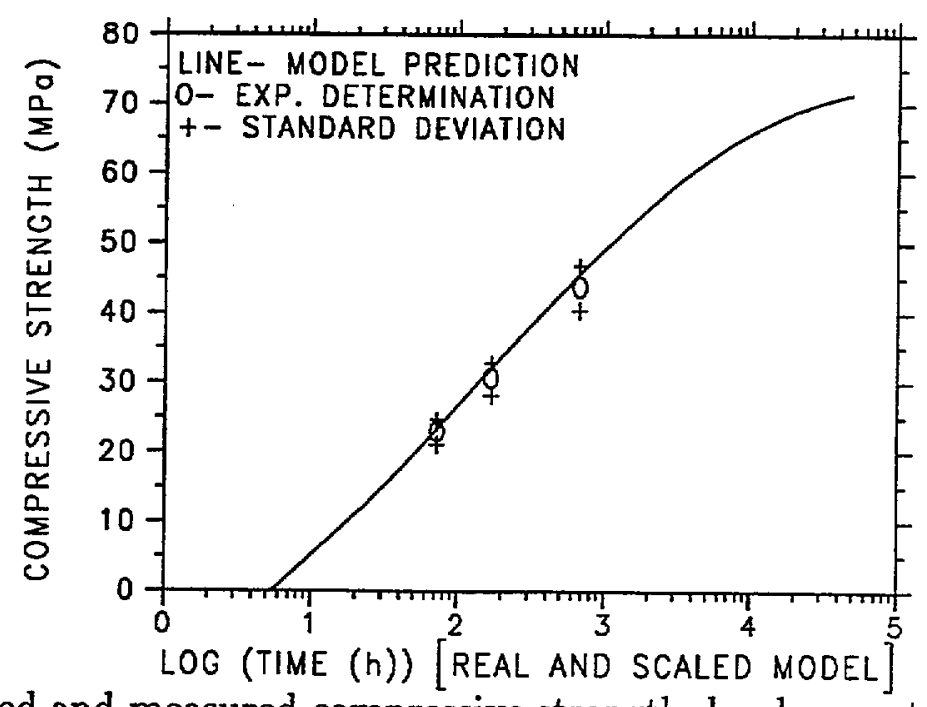

Figure 14: Predicted and measured compressive strength development for Cement 115.

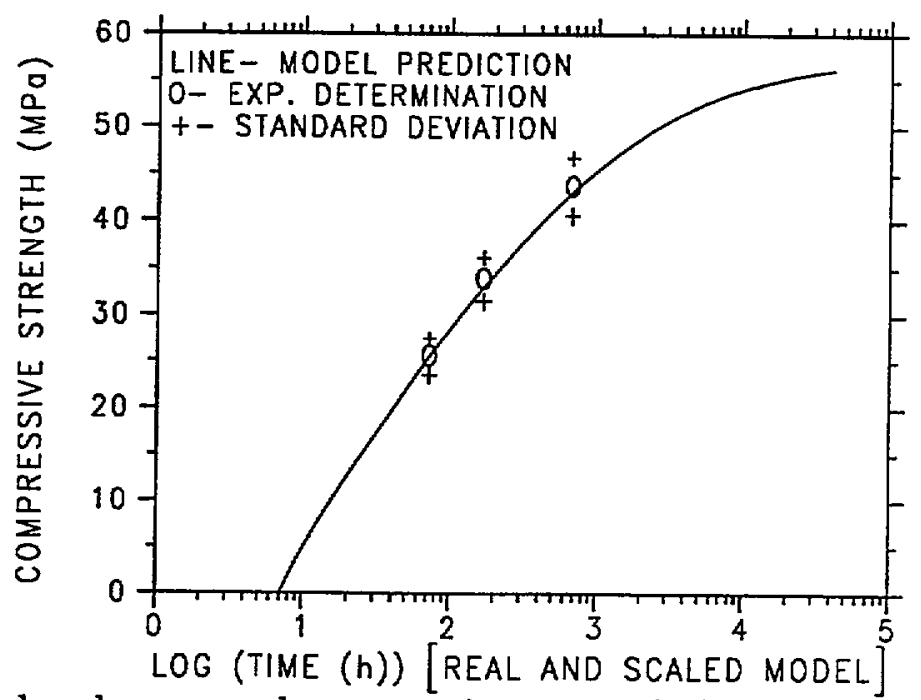

Figure 15: Predicted and measured compressive strength development for Cement 116.

The reproducibility of results of the computer model was investigated in two ways. First, given a starting microstructure (Cement 116 with $w / c=0.4$ ), the 3-D hydration model was executed with three different random number seeds. As can be seen in Fig. 44 in Appendix $\mathrm{C}$, the variability is totally negligible as it is not possible to distinguish the three different degree of hydration curves one from another. In the second case, three different random 
starting microstructures were generated for Cement 116 with $w / c=0.4$. Then, the hydration model was executed on each using the same starting random number seed. Once again, the variability observed in Fig. 45 in Appendix $C$ is seen to be minimal. Thus, matching experimental results to a single execution of the model seems reasonable for calibration purposes.

These results are preliminary in nature as only two cements at room temperature have been studied. However, the results are encouraging, with good agreement between model and experimental results. Ongoing experimental studies are extending the research to two other temperatures $\left(15\right.$ and $35^{\circ} \mathrm{C}$ ) so that an Arrhenius-type model for representing the hydration of cements over a range of temperatures may be investigated. Thus, the coefficients in the parabolic dispersion model of Knudsen will be written as functions of temperature using the Arrhenius equation or an alternative [35]. The model already includes the information necessary for incorporating silica fume into cement-based materials. Here, it will be necessary to calibrate the relative rate of the pozzolanic reaction to that of $C H$ dissolution/precipitation to properly model the observed build up and decay of solid $C H$ volume with time [44]. Preliminary studies have shown that this type of behavior can be achieved in the monophase $C_{3} S$ model system.

\section{Conclusions}

A computer model for representing the hydration and three-dimensional microstructure development of portland cements has been developed. Techniques have been developed for creating three-dimensional starting microstructures which match the particle size distribution, phase volume fractions, and phase surface fractions of a given cement. The model kinetics have been calibrated against experimental measurements using the parabolic dispersion model of Knudsen. For Cements 115 and 116 issued by the Cement and Concrete Reference Laboratory at NIST, it appears that a single simple equation can be used to convert model cycles to real time. After this conversion, the agreements between model and experimental degrees of hydration, heat release, and chemical shrinkage are quite good for the three $w / c$ ratios examined in this study. The model in combination with the gel-space ratio theory of Powers and Brownyard has been successfully applied to predicting the 7 and 28-day compressive strength of ASTM C109 mortar cubes from their 3-day values. While particle size distribution is critical to the successful prediction of cement performance, these results indicate that phase volumes and distributions also have a significant influence on the hydration process, particularly at times exceeding a few days.

\section{Acknowledgments}

The author would like to thank Paul Stutzman of BFRL (NIST) for acquiring the twodimensional images of the CCRL cements, Wei-Guo Lei of the University of Illinois for measuring their particle size distributions, Robin Haupt of CCRL for supplying samples of the cements and results collected from their proficiency sample program, Ken Snyder of BFRL for useful discussions and assistance with the non-evaporable water content measurements, Dr. Eric Lagergren of CAML (NIST) for advice on calibration of the model to experimental results, and Dr. Torben Knudsen of the Technical University of Denmark for advice and suggestions. 


\section{References}

[1] Hill Jr., E.D., and Frohnsdorff, G.J., Cement, Concrete, and Aggregates, 15 (2), 109-118, 1993.

[2] Garboczi, E.J., and Bentz, D.P., MRS Bulletin, 28 (3), 50-54, 1993.

[3] Bentz, D.P., and Garboczi, E.J., "Guide to Using HYDRA3D: A Three-Dimensional Digital-Image-Based Cement Microstructural Model," NISTIR 4746, U.S. Department of Commerce, Jan. 1992.

[4] Bentz, D.P., and Garboczi, E.J., Cement and Concrete Research, 21, 325-344, 1991.

[5] Cement and Concrete Reference Laboratory Proficiency Sample Program: Final Report Portland Cement Proficiency Samples Number 115 and Number 116, Cement and Concrete Reference Laboratory, March, 1995.

[6] Annual Book of ASTM Standards, Vol. 04.01. Cement; Lime; Gypsum (ASTM, Philadelphia, PA, 1992).

[7] Bentz, D.P., and Stutzman, P.E., "SEM Analysis and Computer Modelling of Hydration of Portland Cement Particles," in Petrography of Cementitious Materials, edited by S.M. DeHayes and D. Stark (ASTM, Philadelphia, PA, 1994) p. 60.

[8] Taylor, H.F.W., Cement Chemistry (Academic Press, London, 1990).

[9] Molina, L., "On Predicting the Influence of Curing Conditions on the Degree of Hydration," CBI Report 5:92 (Swedish Cement and Concrete Research Institute, Stockholm, 1992).

[10] Prosen, E.J., Brown, P.W., Frohnsdorff, G., and Davis, R.A., Cement and Concrete Research, 15, 703-710, 1985.

[11] Geiker, M., "Studies of Portland Cement Hydration: Measurements of Chemical Shrinkage and a Systematic Evaluation of Hydration Curves by Means of the Dispersion Model," Ph. D. Thesis, Technical University of Denmark, 1983.

[12] Tazawa, E., Miyazawa, S., and Kasai, T., Cement and Concrete Research, 2, 288-292, 1995.

[13] Hua, C., Acker, P., and Erlacher, A., Bulletin Liaison Laboratoires des Ponts et Chaussees, 196, 79-89, March-April 1995.

[14] Hua, C., Acker, P., and Erlacher, A., Cement and Concrete Research, 25 (7), 1457-1468, 1995.

[15] Bonen, D., and Diamond, S., "Application of Image Analysis to a Comparison of Ball Mill and High Pressure Roller Mill Ground Cement," in Proceedings of the Thirteenth International Conference on Cement Microscopy (International Cement Microscopy Association, 1991) p. 101. 
[16] Bentz, D.P., Garboczi, E.J., and Martys, N.S., "Application of Digital-ImageBased Models to Microstructure, Transport Properties, and Degradation of Cement-Based Materials," Computer Modelling of Microstructure and Its Potential Application to Transport Properties, edited by H.M. Jennings and J. Kropp (Kluwer Academic Pulishers, The Netherlands, 1995).

[17] Jiang, S.P., Mutin, J.C., and Nonat, A., Cement and Concrete Research, 25 (4), 779$789,1995$.

[18] Quiblier, J.A., J. Colloid Interface Sci., 98 (1), 84-102, 1984.

[19] Bentz, D.P., and Martys, N.S., Transport in Porous Media, 17 (3), 221-238, 1995.

[20] Law, A.M., and Kelton, W.D., Simulation Modeling and Analysis (McGraw-Hill, New York, 1982) Chap. 7.

[21] Bentz, D.P., Garboczi, E.J., Pimienta, P.J.P., Carter, W.C., "Cellular Automaton Simulations of Surface Mass Transport Due to Curvature Gradients: Simulation of Sintering," in Synthesis and Processing of Ceramics: Scientific Issues, 249 (Materials Research Society, Pittsburgh, PA, 1992) pp. 413-418.

[22] Bullard, J.W., Garboczi, E.J., Carter, W.C., and Fuller, E.R., Comp. Mat. Sci., 4, 1-14, 1995.

[23] Bentz, D.P., Coveney, P.V., Garboczi, E.J., Kleyn, M.F., and Stutzman, P.E., Modelling and Simulation in Materials Science and Engineering, 2, 783-808, 1994.

[24] Mindess, S., and Young, J.F., Concrete (Prentice-Hall, Englewood, NJ, 1981).

[25] Young, J.F., and Hansen, W., "Volume Relationship for C-S-H Formation Based on Hydration Stoichiometry," in Microstructural Development During Hydration of Cement, 235 (Materials Research Society, Pittsburgh, PA, 1986) pp. 313-322.

[26] Fukuhara, M., Goto, S., Asaga, K., Daimon, M., and Kondo, R., Cement and Concrete Research, 11, 407-414, 1981.

[27] Handbook of Chemistry and Physics, 63rd ed. (CRC Press, Boca Raton, FL, 1982).

[28] Schwarz, W., Journal of Advanced Cement-Based Materials, 2, 189-200, 1995.

[29] Jennings, H.M., and Parrott, L.J., Journal of Materials Science, 21, 4053, 1986.

[30] Bentz, D.P., Martys, N.S., Stutzman, P.E., Levenson, M.S., Garboczi, E.J., Dunsmuir, J., and Schwartz, L.M., "X-ray Microtomography of an ASTM C109 Mortar Exposed to Sulfate Attack," in Microstructure of Cement-Based Systems/ Bonding and Interfaces in Cementitious Materials, 370 (Materials Research Society, Pittsburgh, PA, 1995) pp. 77-82.

[31] Tzschichholz, F., Herrmann, H.J., and Zanni, H., "A Reaction-Diffusion Model for the Hydration/Setting of Cement", submitted to Phys. Rev. E.

[32] Parrott, L.J., Geiker, M., Gutteridge, W.A., Killoh, D., Cement and Concrete Research, 20, 919-926, 1990. 
[33] Powers, T.C., Industrial and Engineering Chemistry, 27, 790-794, 1935.

[34] Olson, R.A., Christensen, B.J., Coverdale, R.T., Ford, S.J., Moss, G.M., Jennings, H.M., Mason, T.O., and Garboczi, E.J., "Interpretation of the Impedance Spectroscopy of Cement Paste via Computer Modelling: Part III. Microstructural Analysis of Frozen", Journal of Materials Science, 30, 5078-5086, 1995.

[35] Carino, N.J., Knab, L.I., and Clifton, J.R., "Applicability of the Maturity Method to High-Performance Concrete," NISTIR 4819, U.S. Department of Commerce, May 1992.

[36] Knudsen, T., Cement and Concrete Research, 14, 622-630, 1984.

[37] Filliben, J.J., "DATAPLOT: Introduction and Overview", NBS Special Publication 667, U.S. Department of Commerce, 1984.

[38] Brown, P.W., Journal of the American Ceramic Society, 76 (12), 2971-2976, 1993.

[39] Gaidis, J.M., and Gartner, E.M., "Hydration Mechanisms II", in Materials Science of Concrete II, edited by J.P. Skalny and S. Mindess (American Ceramic Society, Westerville, OH, 1991) p. 9.

[40] Pratt, P.L., and Ghose, A., Phil. Trans. R. Soc. London, A310, 93-103, 1983.

[41] Knudsen, T., personal communication, 1991.

[42] Pommersheim, J.M., "Effect of Particle Size Distribution on Hydration Kinetics," Mat. Res. Soc. Symp. Proc. Vol. 85, (Materials Research Society, Pittsburgh, PA, 1987) p. 301.

[43] Radjy, F.F., and Vunic, D.W., "Heat Signature Testing of Concrete," in Proceedings of Structural Materials Technology- An NDT Conference, Atlantic City, 1994.

[44] Lu, P., Sun, G.K., and Young, J.F., Journal of the American Ceramic Society, 76, 1003-1007, 1993. 


\section{Appendices}

\section{A Graphs for Fit of Knudsen's Dispersion Models to Experimental Results}
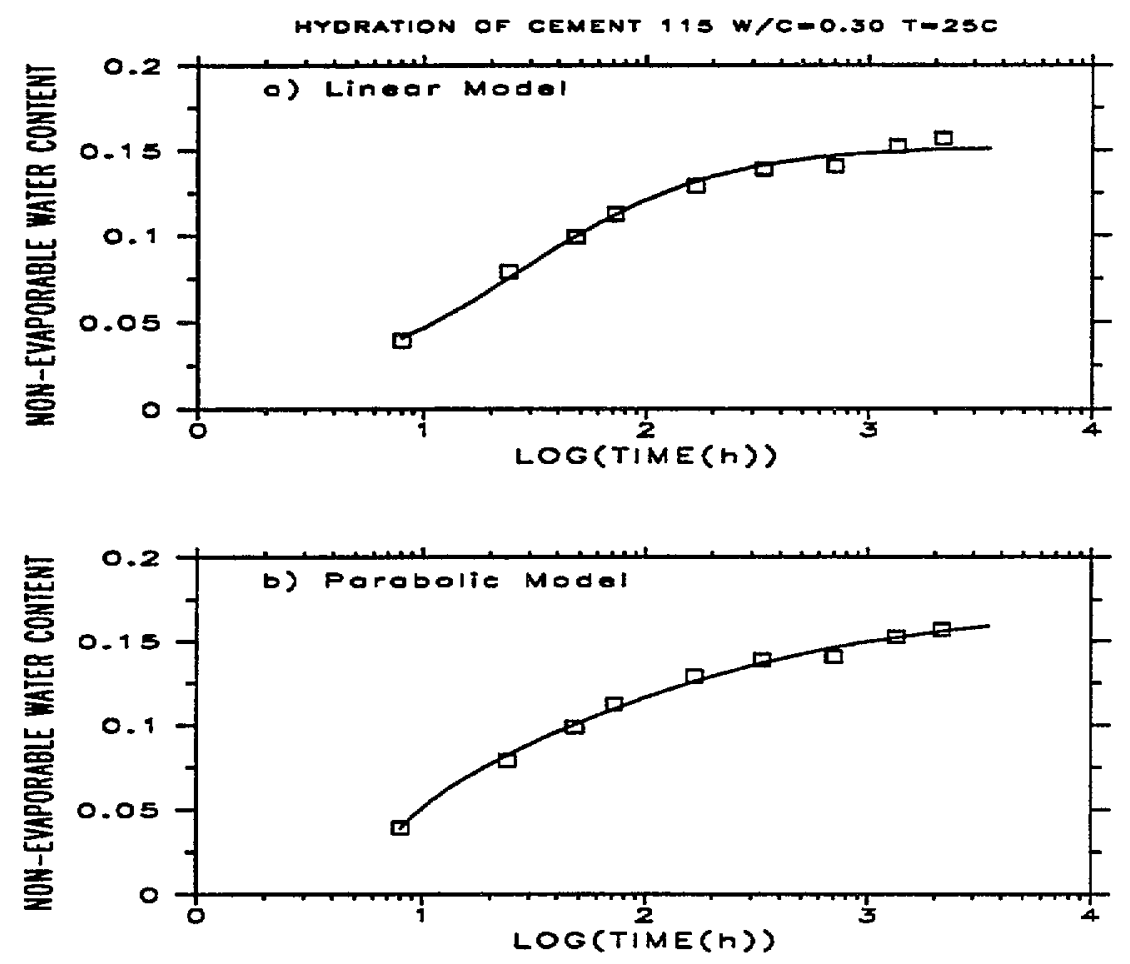

Figure 16: Fits of Knudsen's dispersion models to experimental non-evaporable water content vs. time for Cement 115 and $w / c=0.3$. 

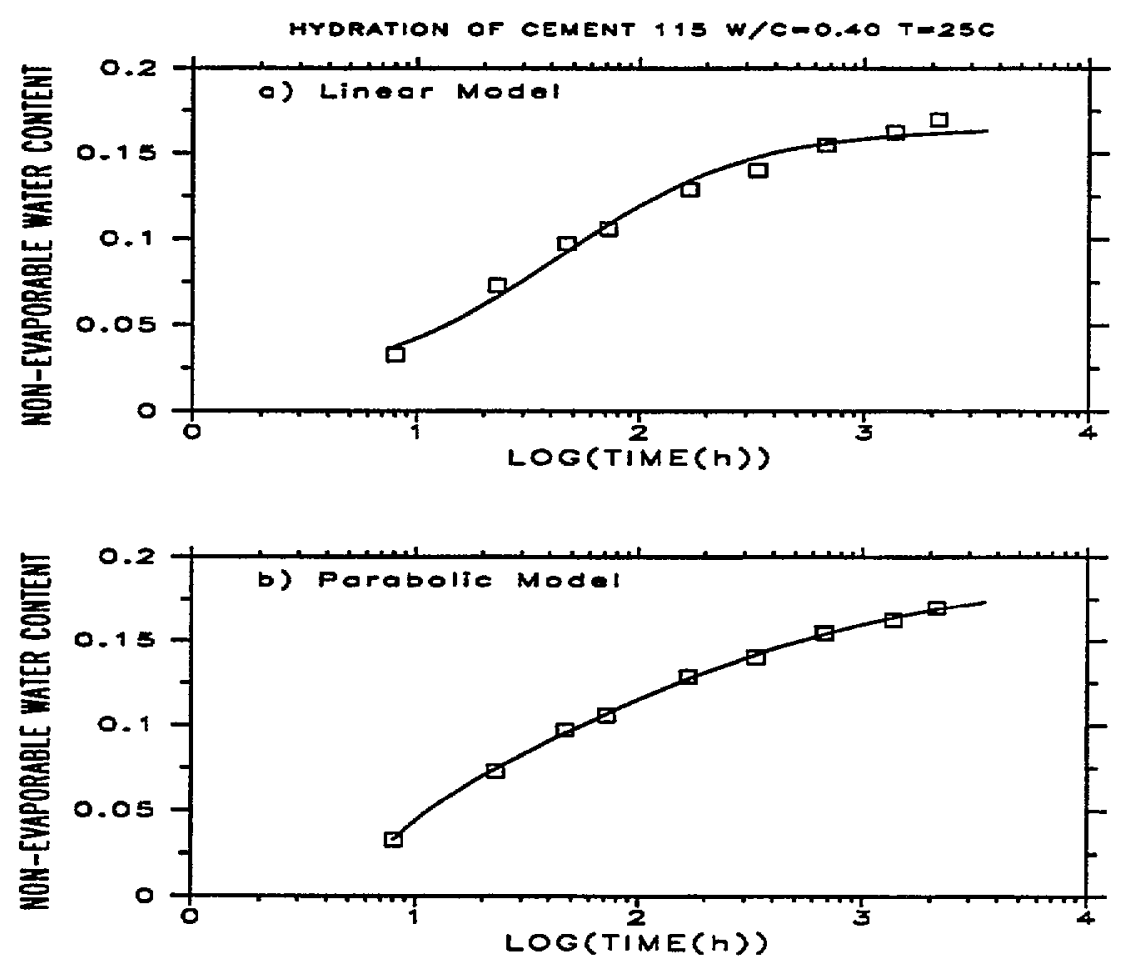

Figure 17: Fits of Knudsen's dispersion models to experimental non-evaporable water content vs. time for Cement 115 and $w / c=0.4$.
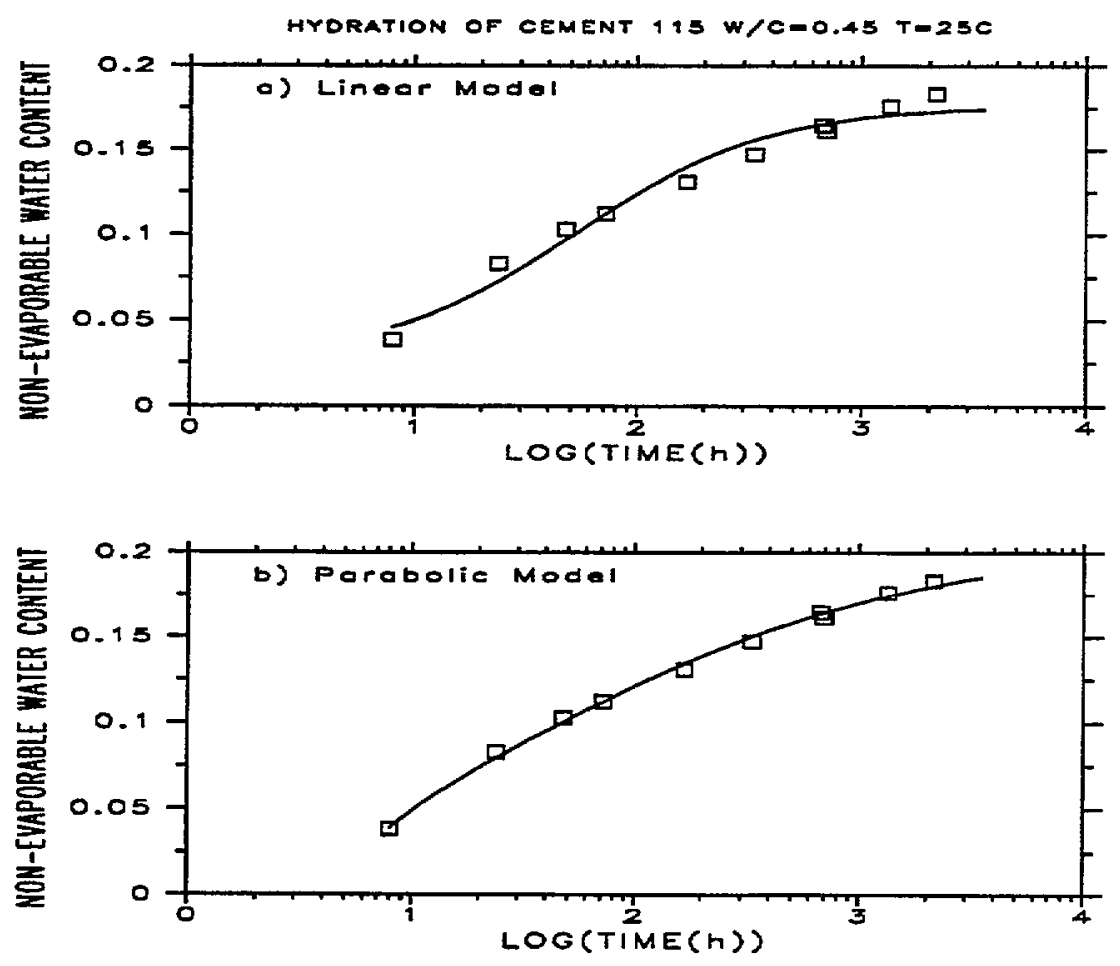

Figure 18: Fits of Knudsen's dispersion models to experimental non-evaporable water content vs. time for Cement 115 and $w / c=0.45$. 

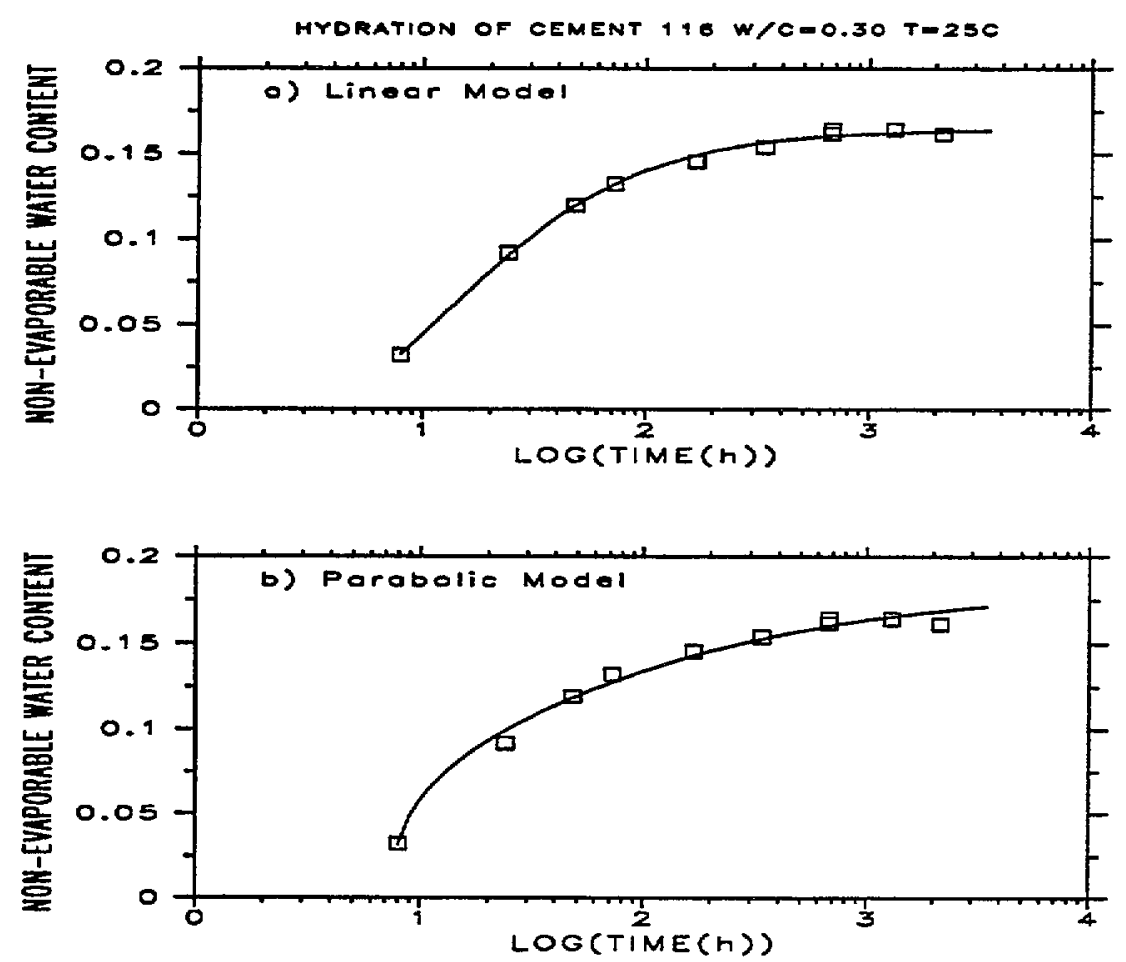

Figure 19: Fits of Knudsen's dispersion models to experimental non-evaporable water content vs. time for Cement 116 and $w / c=0.3$.
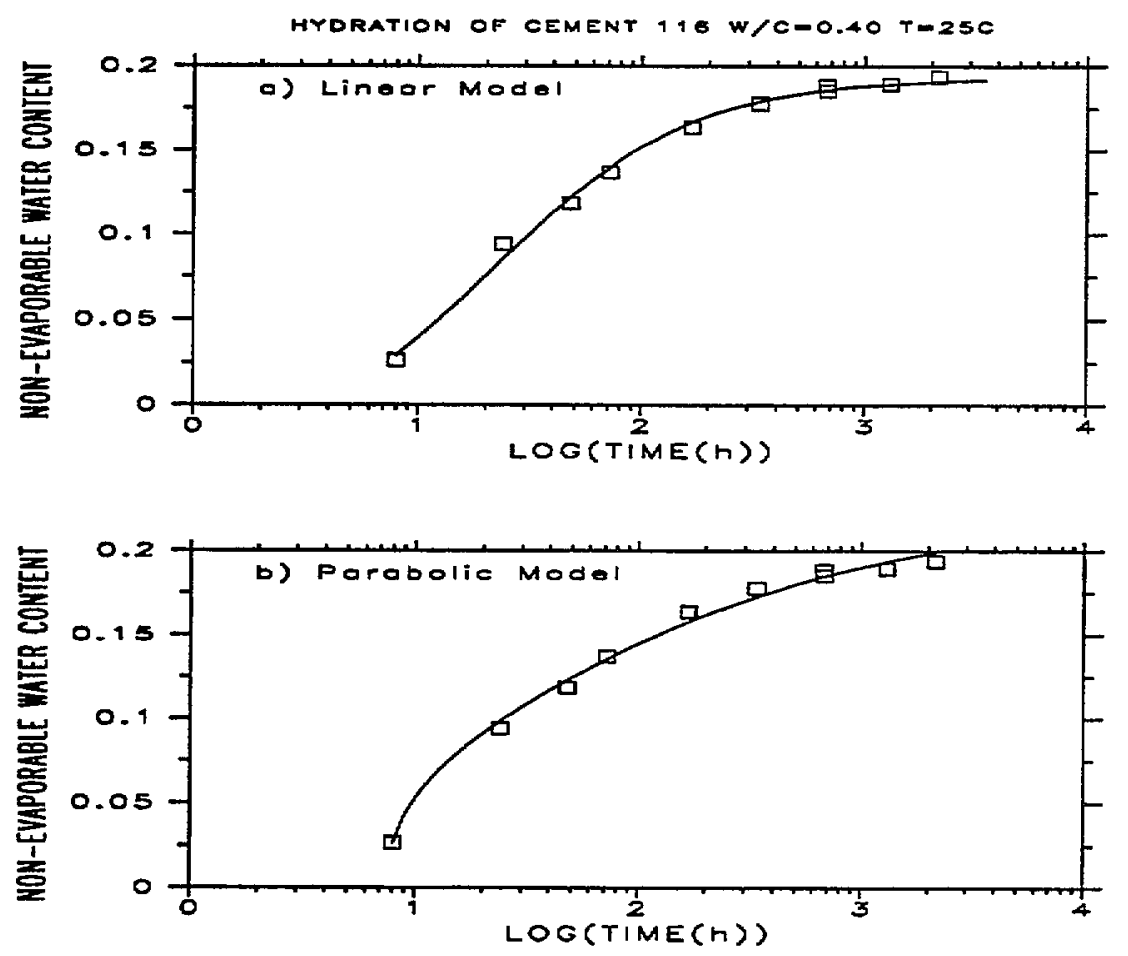

Figure 20: Fits of Knudsen's dispersion models to experimental non-evaporable water content vs. time for Cement 116 and $w / c=0.4$. 

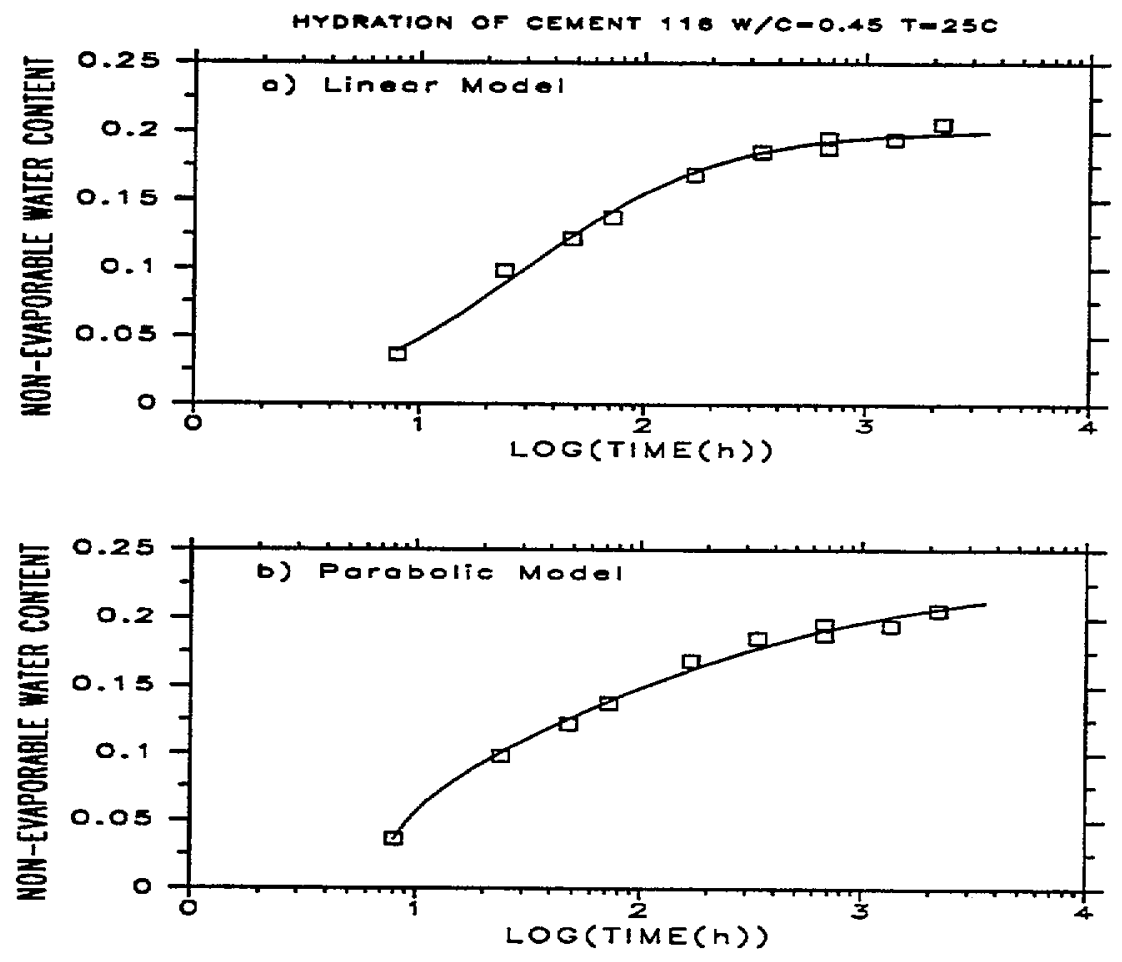

Figure 21: Fits of Knudsen's dispersion models to experimental non-evaporable water content vs. time for Cement 116 and $w / c=0.45$. 


\section{B Model vs. Experimental Results}

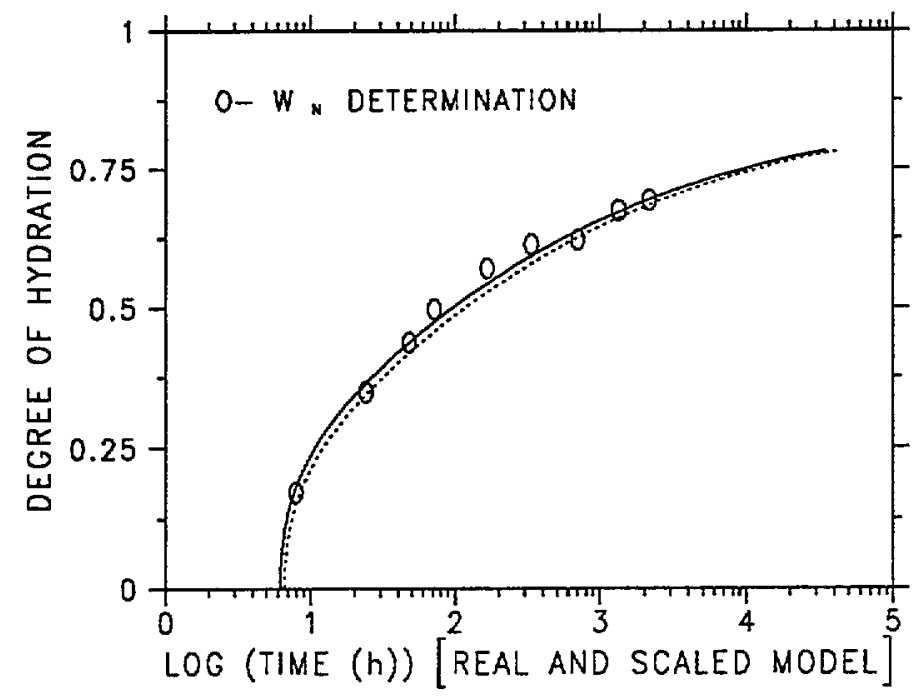

Figure 22: Measured and model degree of hydration vs. time for Cement 115 with $w / c=0.30$.

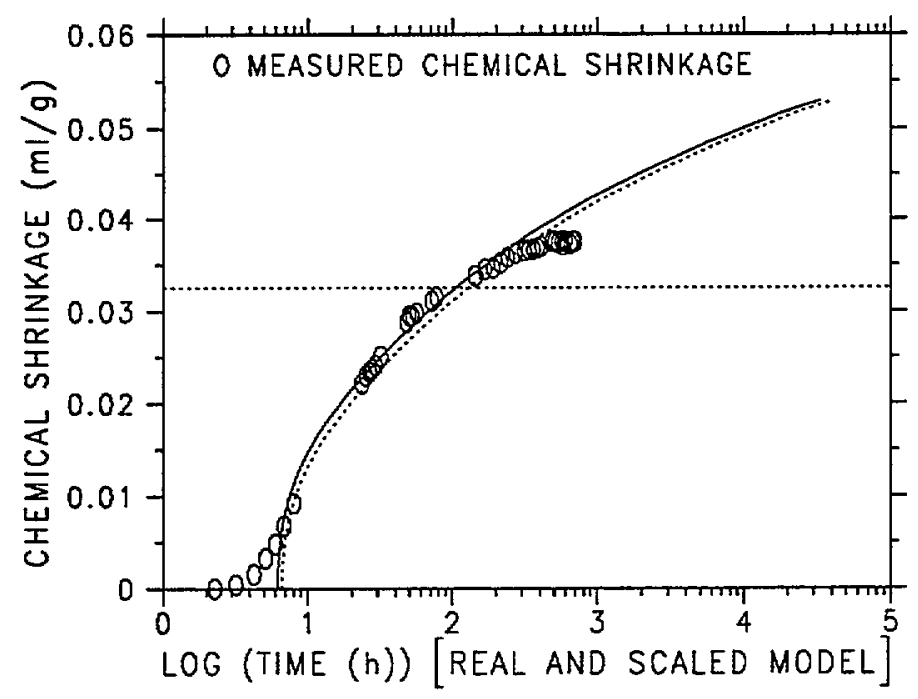

Figure 23: Measured and model chemical shrinkage vs. time for Cement 115 with $w / c=0.30$. 


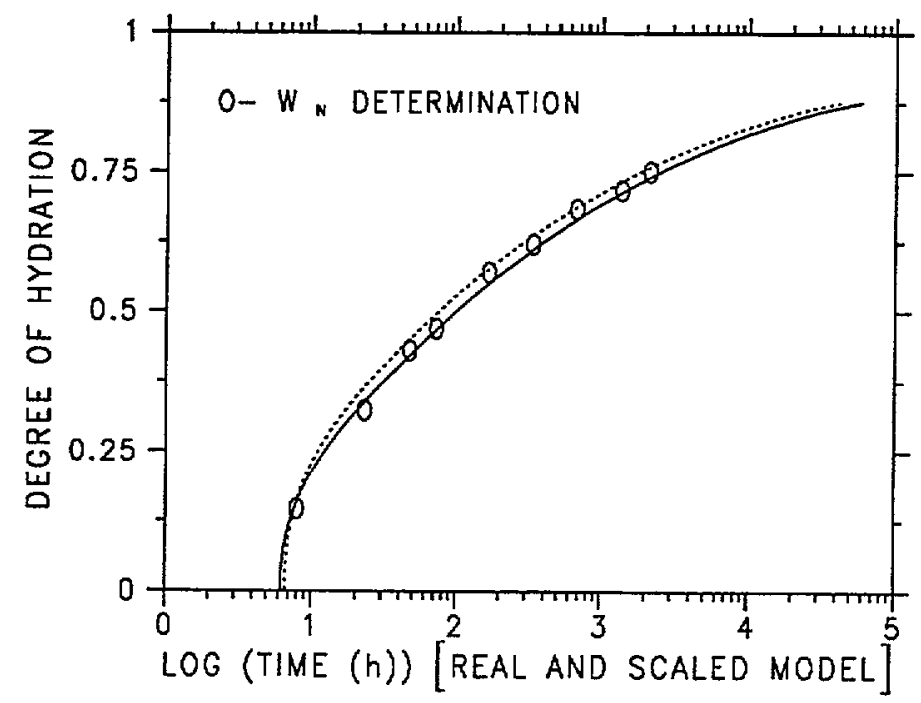

Figure 24: Measured and model degree of hydration vs. time for Cement 115 with $w / c=0.40$.

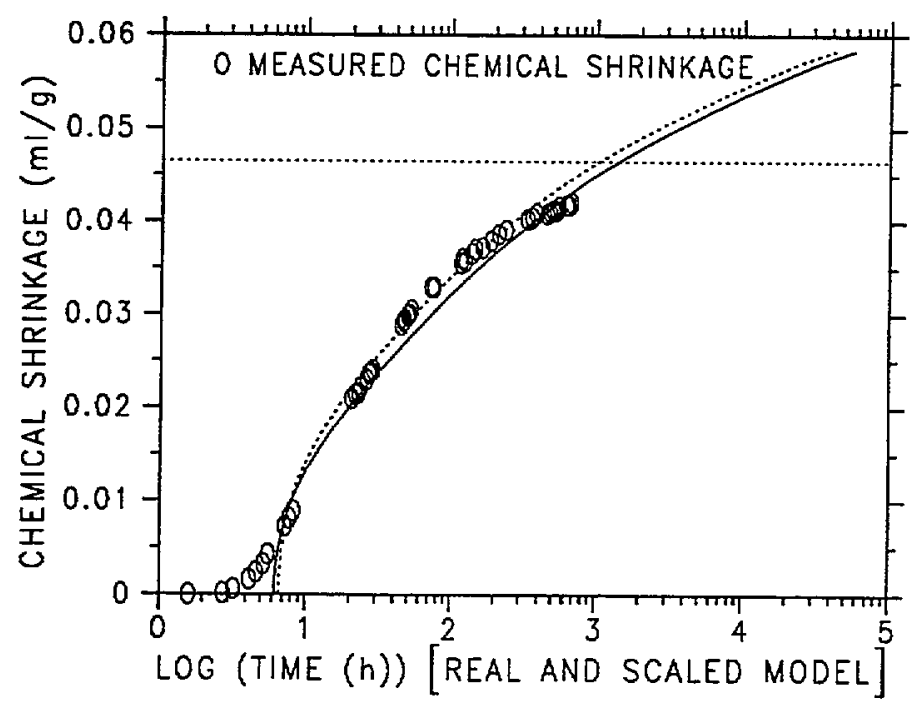

Figure 25: Measured and model chemical shrinkage vs. time for Cement 115 with $w / c=0.40$. 


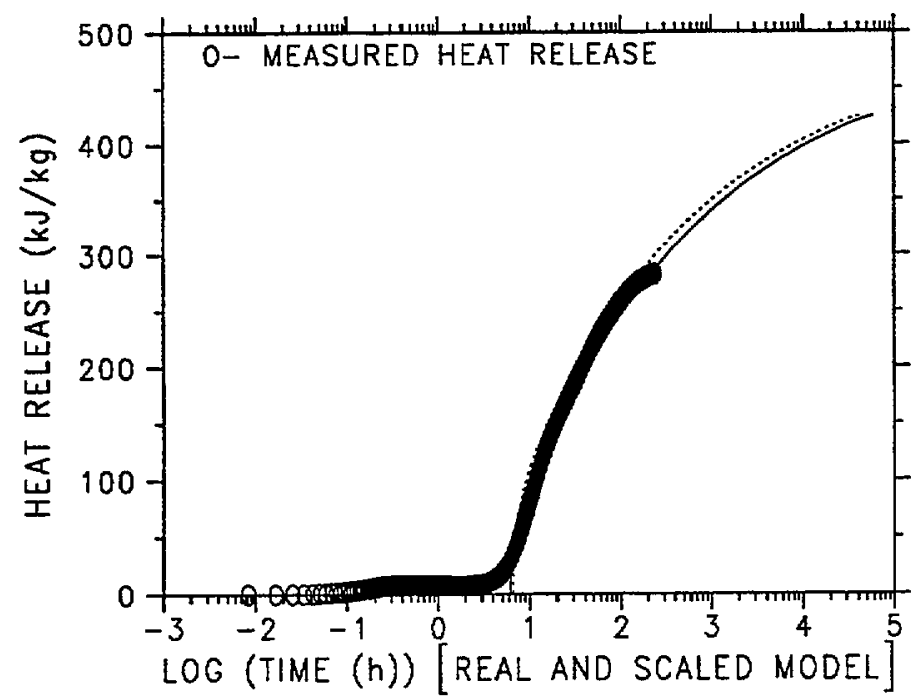

Figure 26: Measured and model heat release vs. time for Cement 115 with $w / c=0.40$.

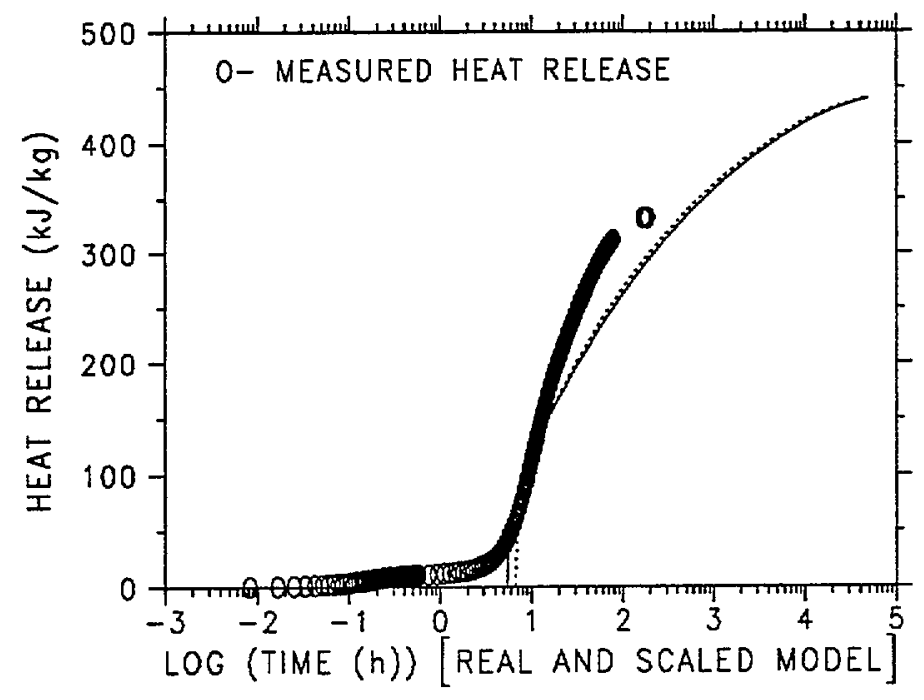

Figure 27: Measured and model heat release vs. time for Cement 115 with $w / c=0.45$. 


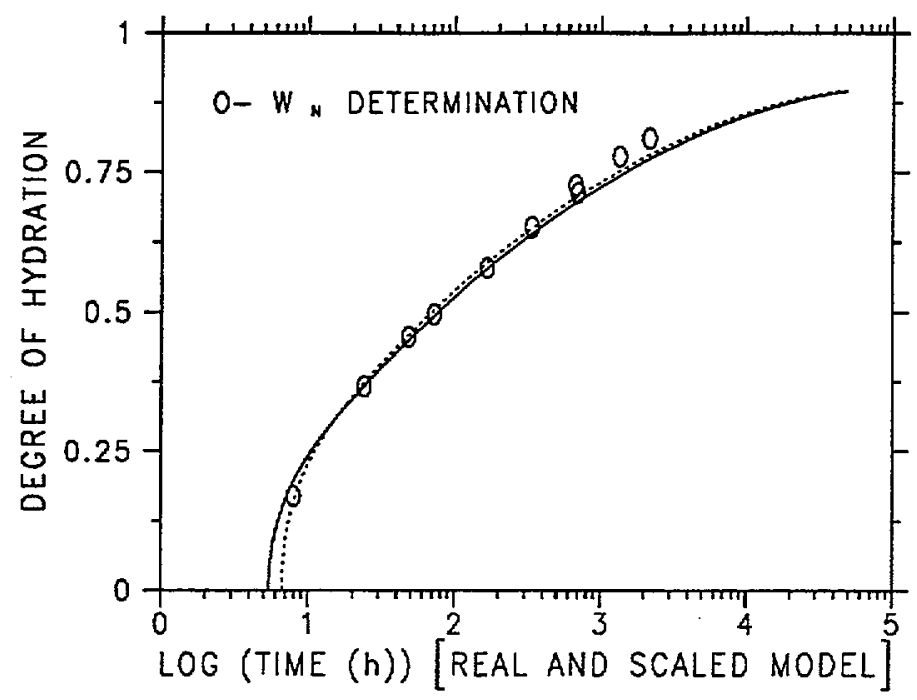

Figure 28: Measured and model degree of hydration vs. time for Cement 115 with $w / c=0.45$.

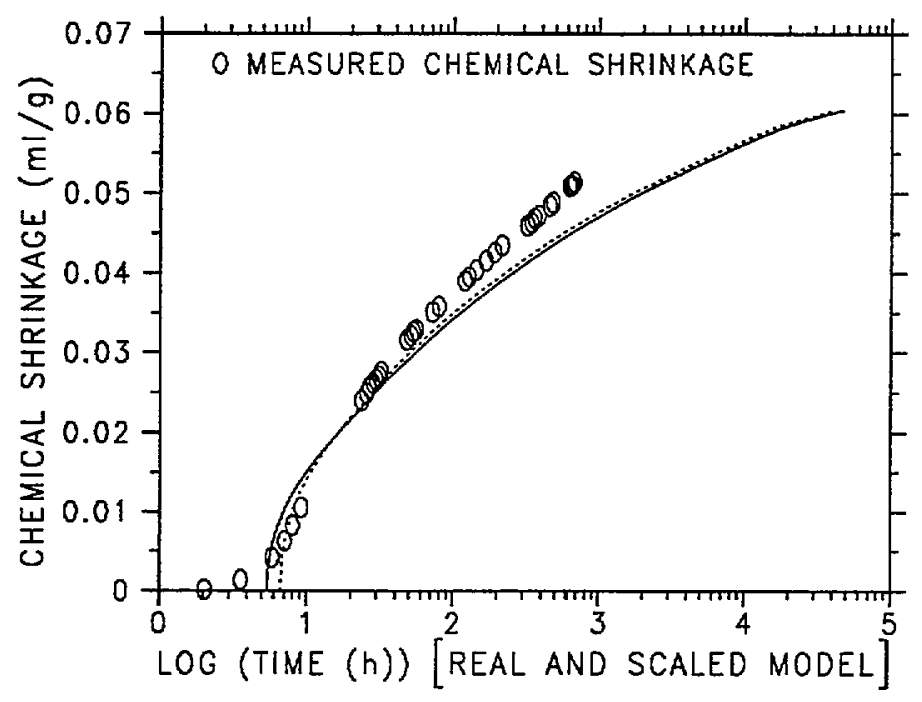

Figure 29: Measured and model chemical shrinkage vs. time for Cement 115 with $w / c=0.45$. 


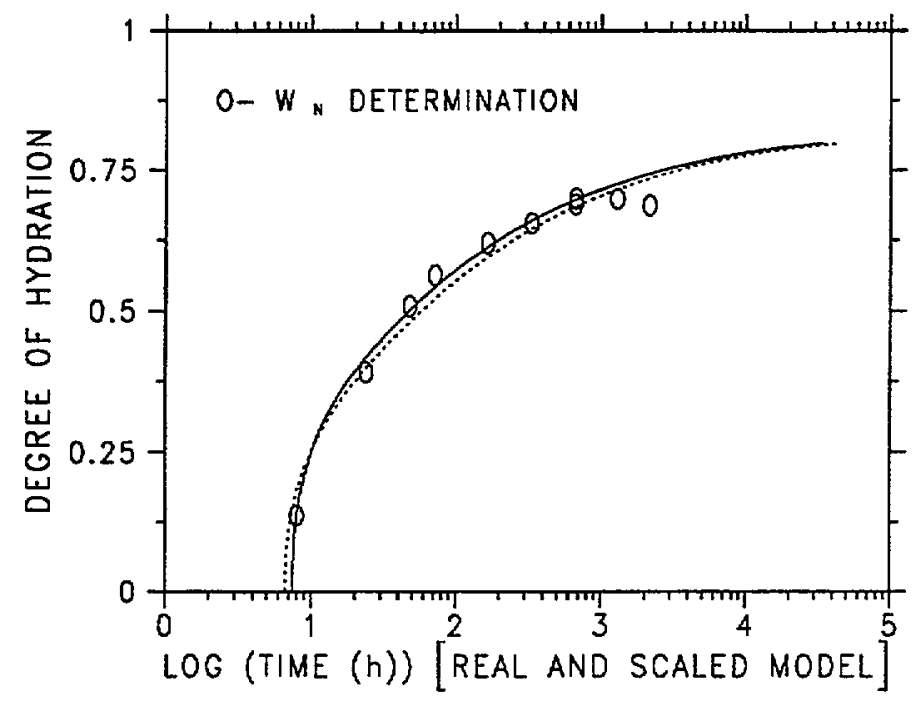

Figure 30: Measured and model degree of hydration vs. time for Cement 116 with $w / c=0.30$.

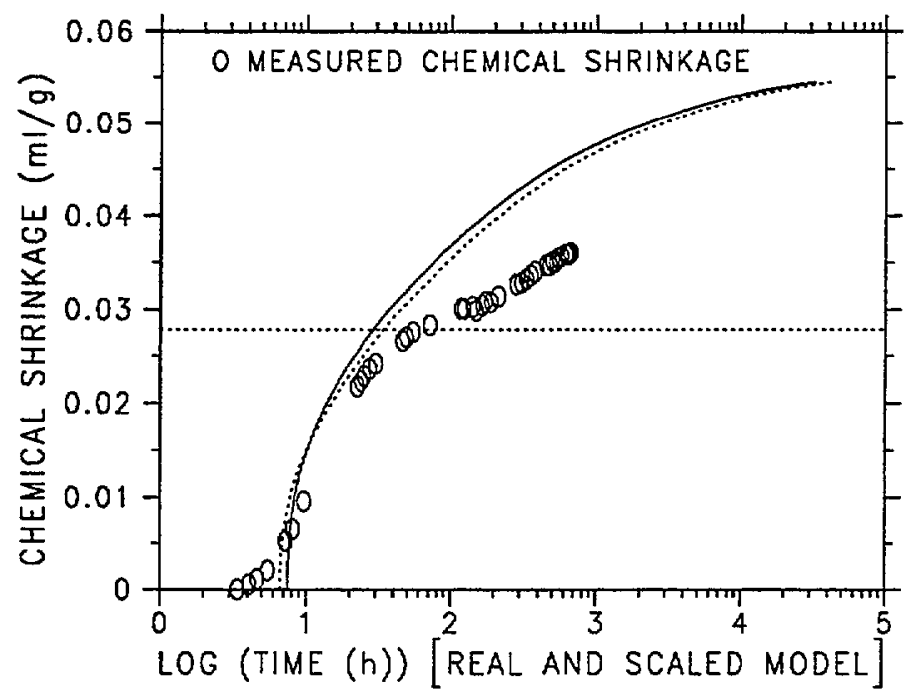

Figure 31: Measured and model chemical shrinkage vs. time for Cement 116 with $w / c=0.30$. 


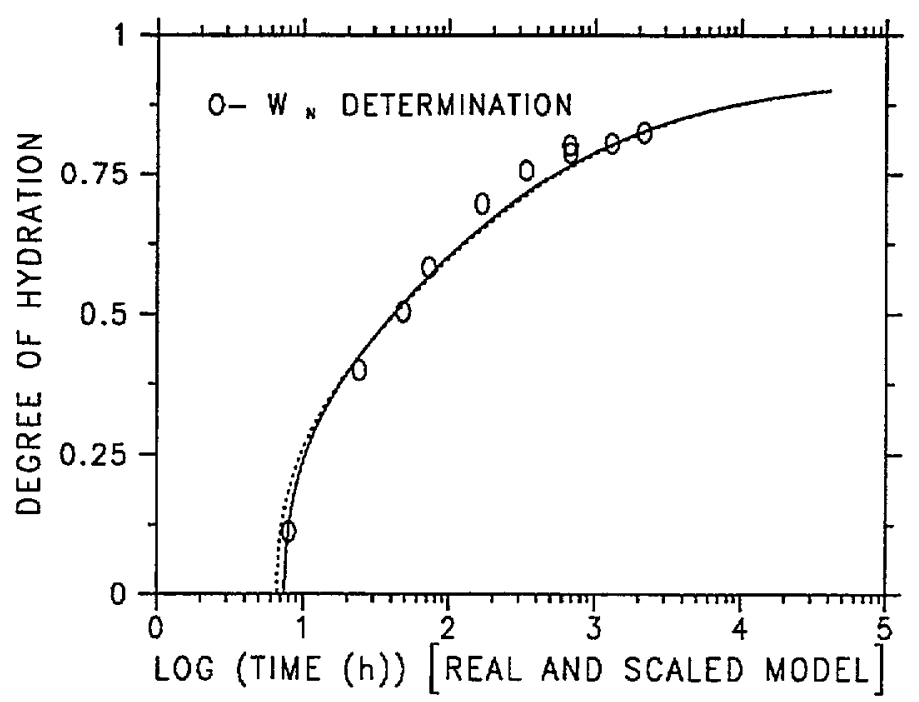

Figure 32: Measured and model degree of hydration vs. time for Cement 116 with $w / c=0.40$.

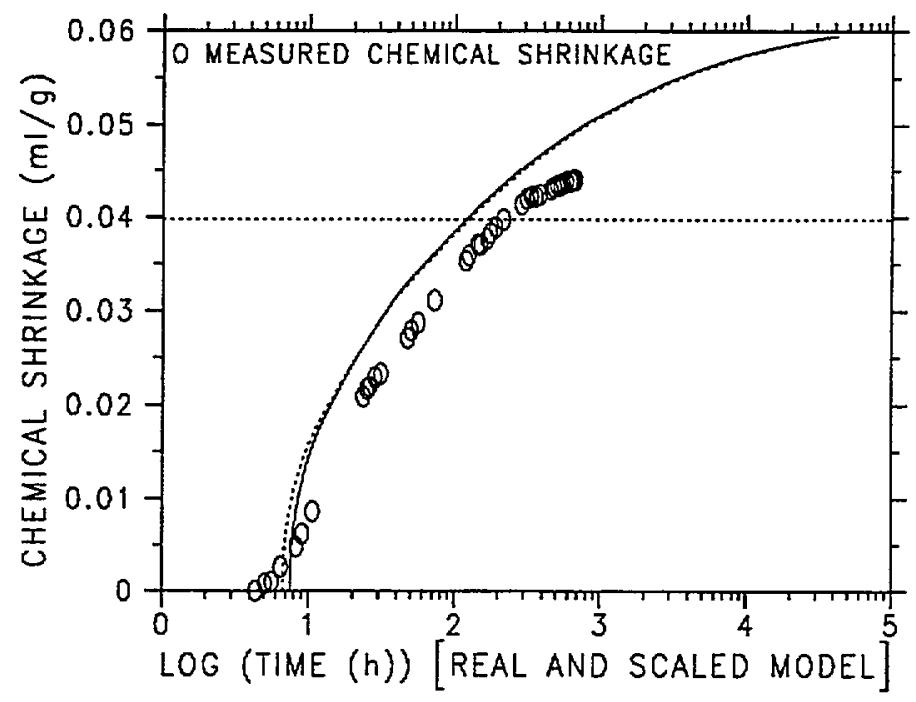

Figure 33: Measured and model chemical shrinkage vs. time for Cement 116 with $w / c=0.40$. 


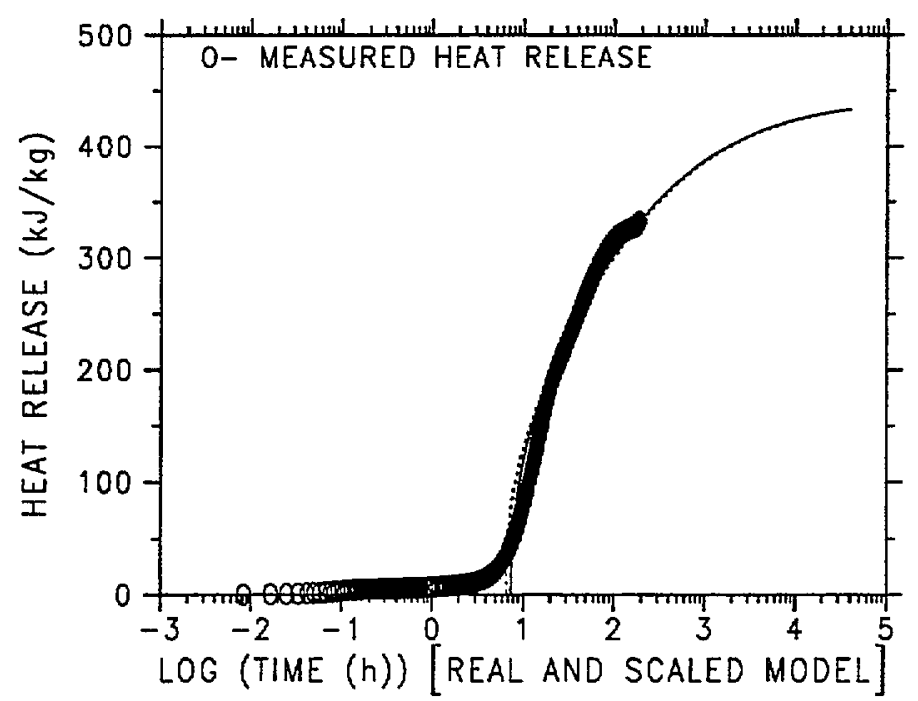

Figure 34: Measured and model heat release vs. time for Cement 116 with $w / c=0.40$.

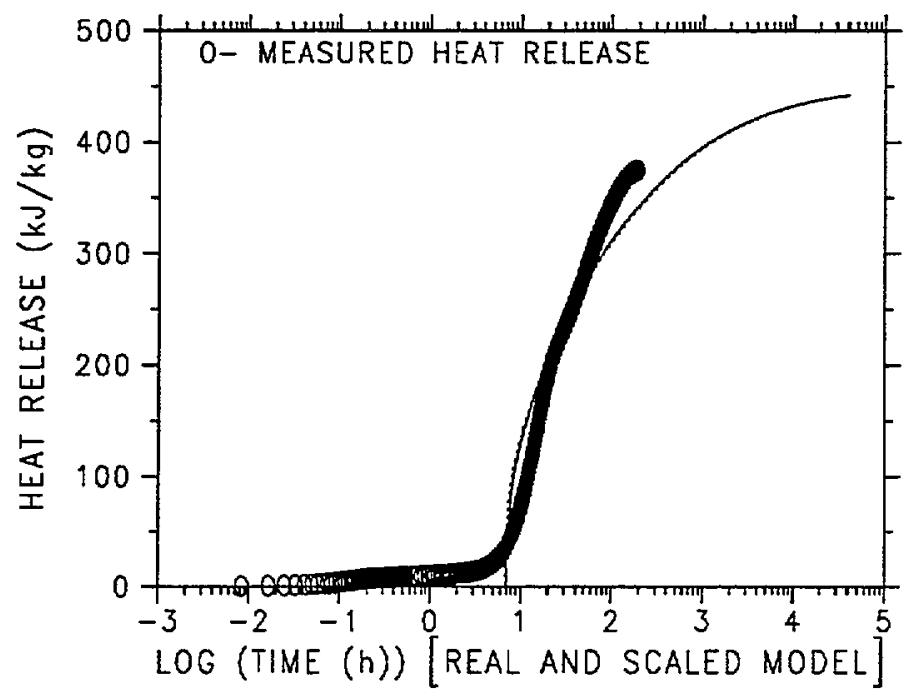

Figure 35: Measured and model heat release vs. time for Cement 116 with $w / c=0.45$. 


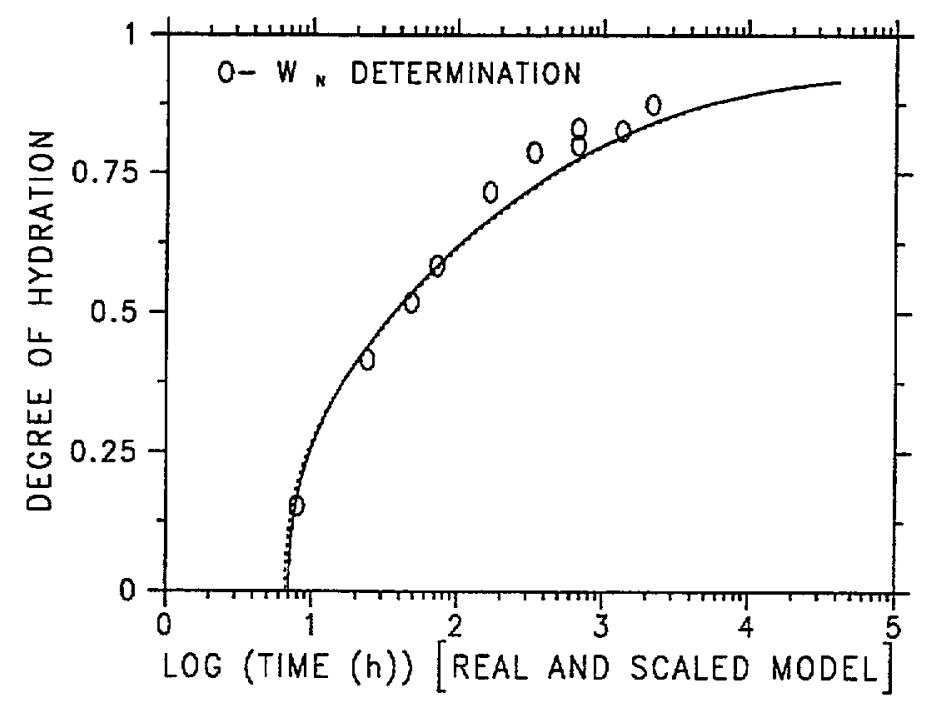

Figure 36: Measured and model degree of hydration vs. time for Cement 116 with $w / c=0.45$.

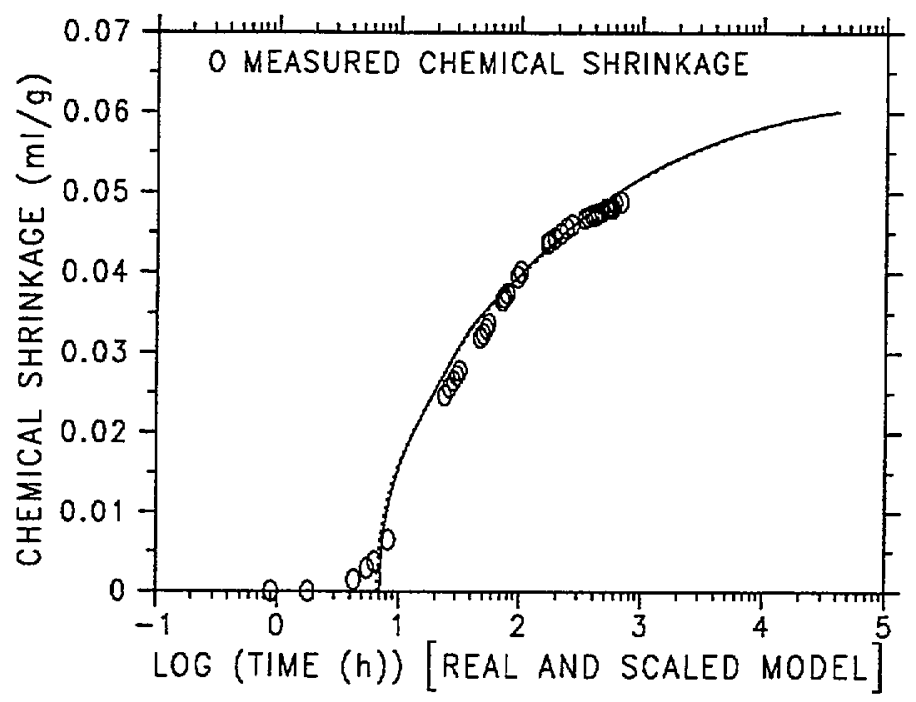

Figure 37: Measured and model chemical shrinkage vs. time for Cement 116 with $w / c=0.45$. 


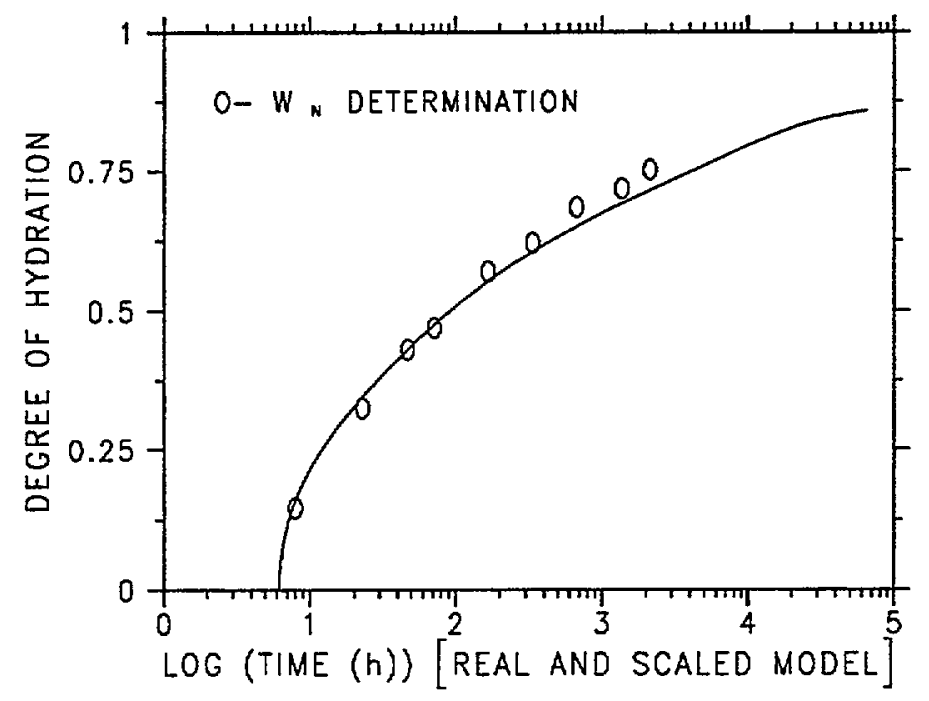

Figure 38: Measured and model degree of hydration vs. time for Cement 115 with $w / c=0.40$ for monophase Bogue representation.

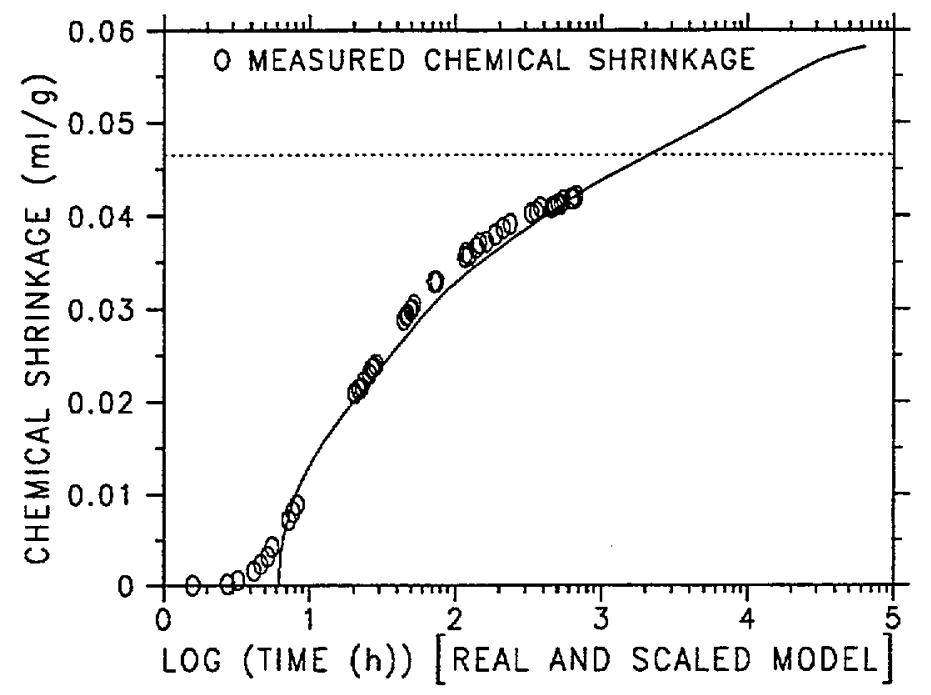

Figure 39: Measured and model chemical shrinkage vs. time for Cement 115 with $w / c=0.40$ for monophase Bogue representation. 


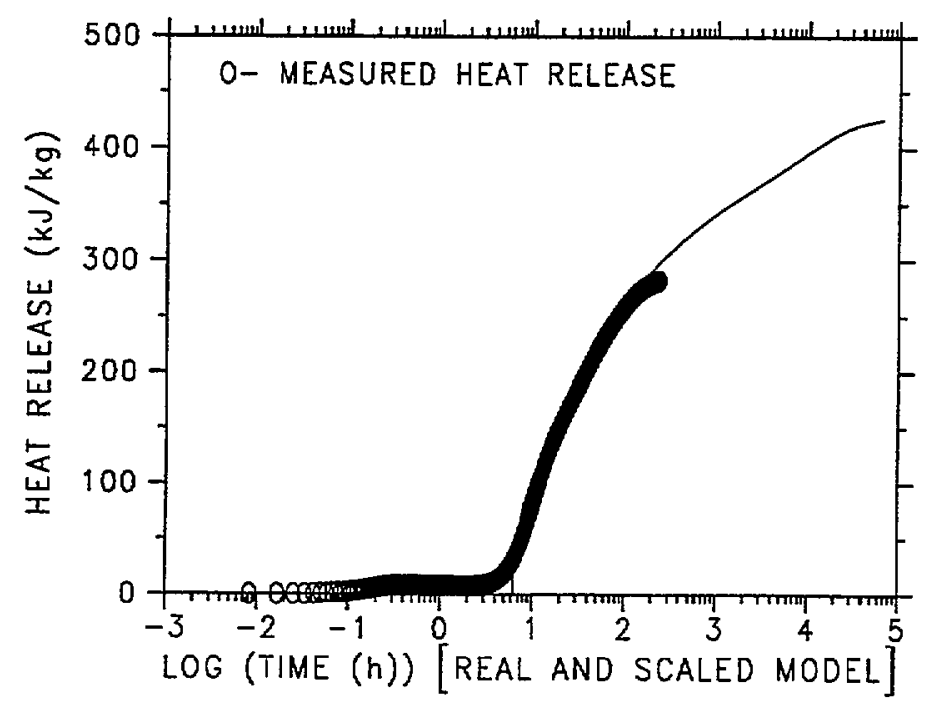

Figure 40: Measured and model heat release vs. time for Cement 115 with $w / c=0.40$ for monophase Bogue representation.

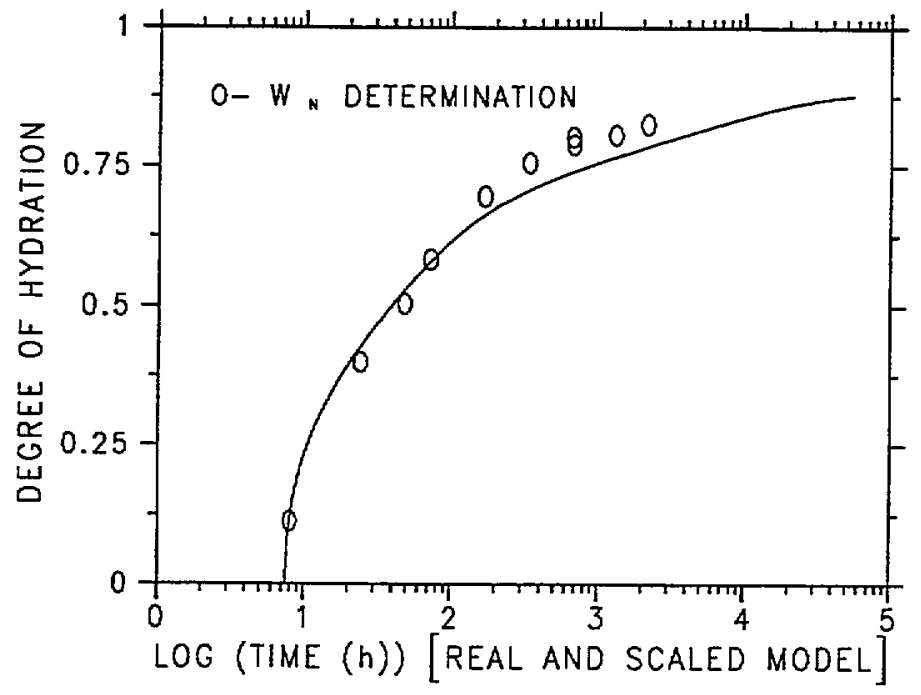

Figure 41: Measured and model degree of hydration vs. time for Cement 116 with $w / c=0.40$ for monophase Bogue representation. 


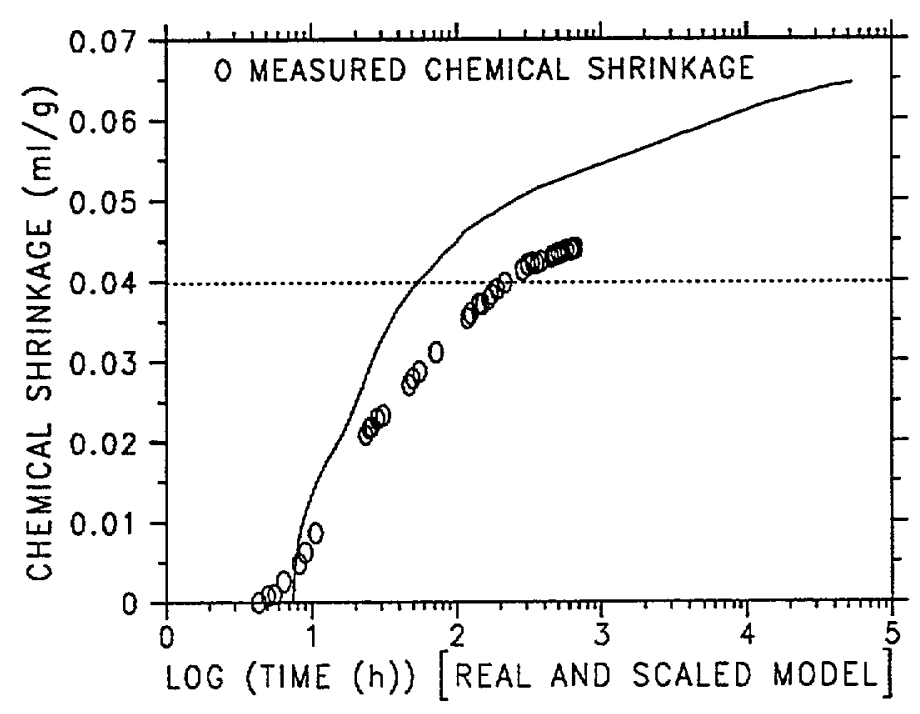

Figure 42: Measured and model chemical shrinkage vs. time for Cement 116 with $w / c=0.40$ for monophase Bogue representation.

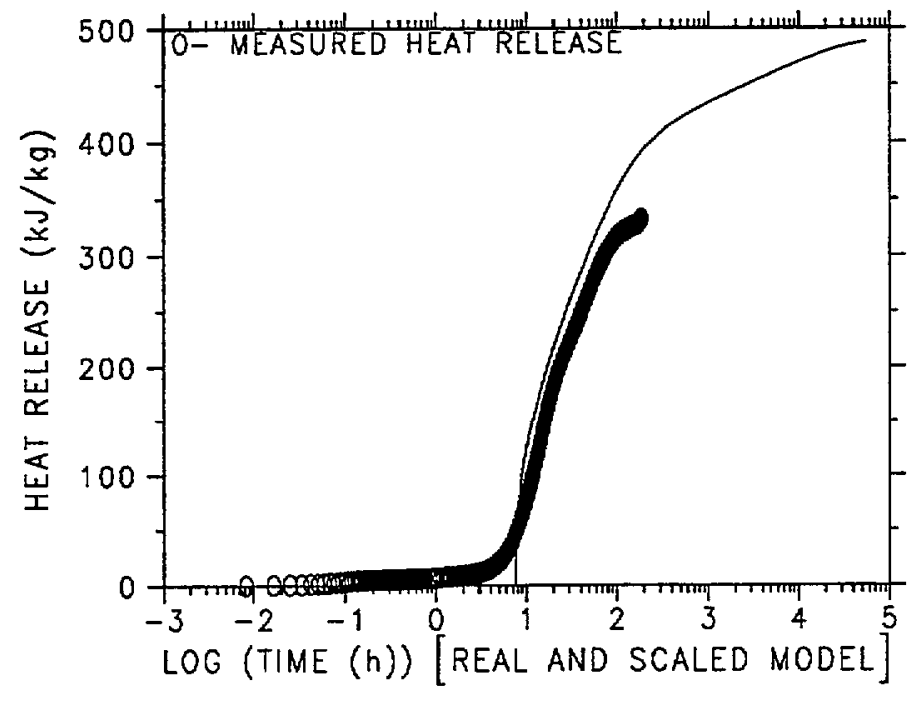

Figure 43: Measured and model heat release vs. time for Cement 116 with $w / c=0.40$ for monophase Bogue representation. 


\section{Variability of Computer Simulation Results}

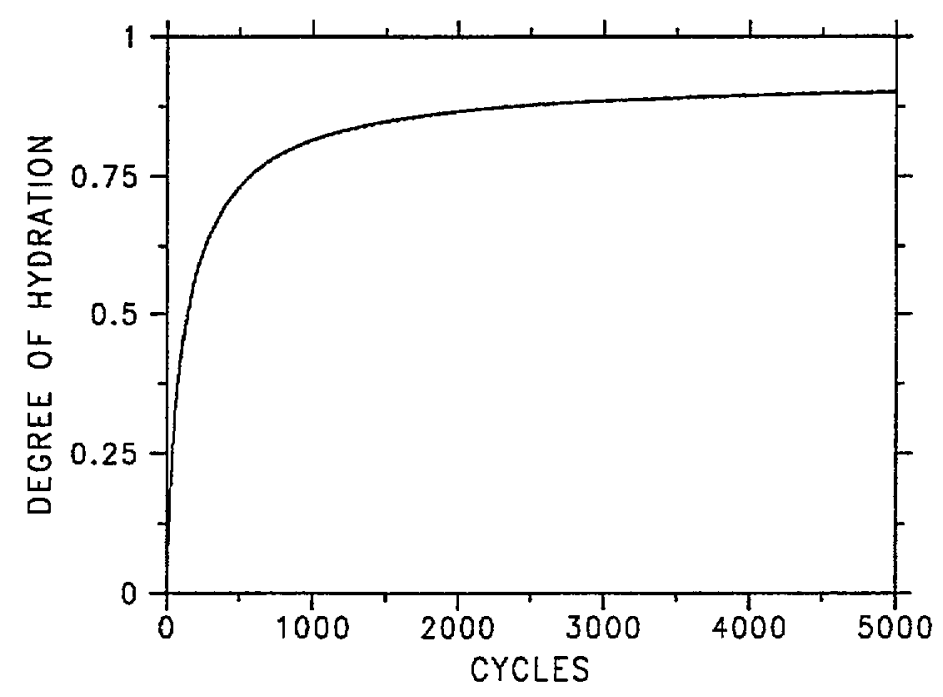

Figure 44: Variation in results for three executions of 3-D hydration model on a single starting microstructure (Cement 116 with $w / c=0.4$ ).

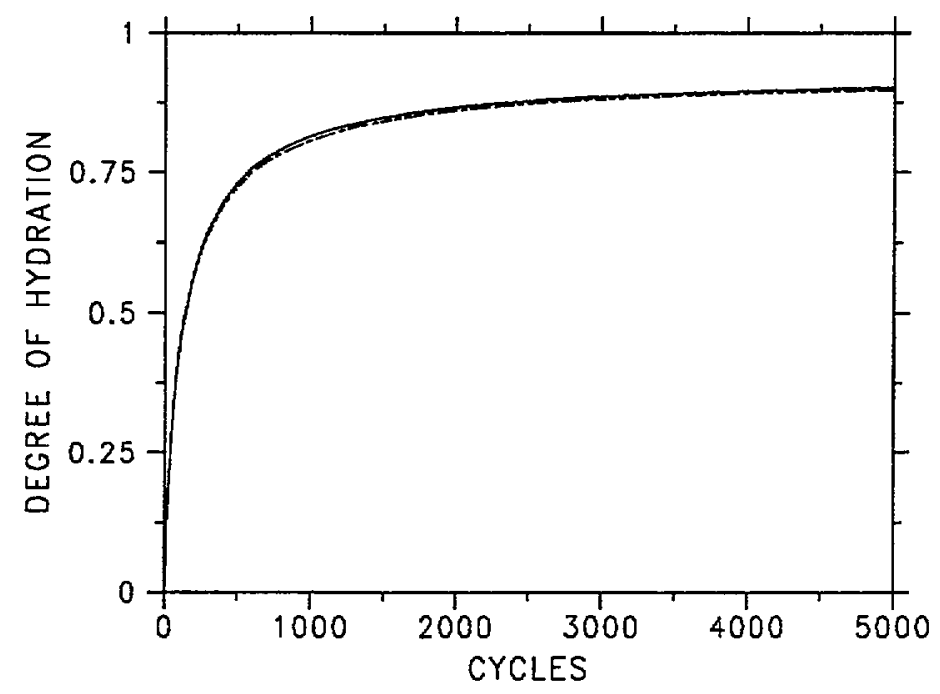

Figure 45: Variation in results for single executions of 3-D hydration model on three different starting microstructures (Cement 116 with $w / c=0.4$ ). 
